\title{
CORE AND FILAMENT FORMATION IN MAGNETIZED, SELF-GRAVITATING ISOTHERMAL LAYERS
}

\author{
Sven Van Loo, Eric Keto, and Qizhou Zhang \\ Harvard-Smithsonian Center for Astrophysics, 60 Garden Street, Cambridge, MA 02138, USA; svanloo@cfa.harvard.edu \\ Received 2014 January 1; accepted 2014 May 16; published 2014 June 12
}

\begin{abstract}
We examine the role of the gravitational instability in an isothermal, self-gravitating layer threaded by magnetic fields on the formation of filaments and dense cores. Using a numerical simulation, we follow the non-linear evolution of a perturbed equilibrium layer. The linear evolution of such a layer is described in the analytic work of Nagai et al. We find that filaments and dense cores form simultaneously. Depending on the initial magnetic field, the resulting filaments form either a spiderweb-like network (for weak magnetic fields) or a network of parallel filaments aligned perpendicular to the magnetic field lines (for strong magnetic fields). Although the filaments are radially collapsing, the density profile of their central region (up to the thermal scale height) can be approximated by a hydrodynamical equilibrium density structure. Thus, the magnetic field does not play a significant role in setting the density distribution of the filaments. The density distribution outside of the central region deviates from the equilibrium. The radial column density distribution is then flatter than the expected power law of $r^{-4}$ and similar to filament profiles observed with Herschel. Our results do not explain the near constant filament width of $\sim 0.1 \mathrm{pc}$. However, our model does not include turbulent motions. It is expected that the accretion-driven amplification of these turbulent motions provides additional support within the filaments against gravitational collapse. Finally, we interpret the filamentary network of the massive star forming complex G14.225-0.506 in terms of the gravitational instability model and find that the properties of the complex are consistent with being formed out of an unstable layer threaded by a strong, parallel magnetic field.
\end{abstract}

Key words: ISM: clouds - ISM: structure - methods: numerical - stars: formation

Online-only material: animations, color figures

\section{INTRODUCTION}

Molecular clouds exhibit a hierarchical density structure with stars forming in the densest regions. Often, these star-forming complexes have an elongated, filamentary shape (e.g., Schneider \& Elmegreen 1979; Johnstone \& Bally 1999; Mizuno et al. 1995; Goldsmith et al. 2008). In fact, recent observations by the Herschel Space Observatory show that parsec-scale filaments are ubiquitous in the interstellar medium (ISM; e.g., André et al. 2010; Men'shchikov et al. 2010; Arzoumanian et al. 2011; Molinari et al. 2011). The filaments tend to extend out from dense star-forming hubs (Myers 2009; Liu et al. 2012; Galván-Madrid et al. 2013) and, sometimes, these hub-filament structures are part of a larger-scale pattern with parallel filaments (Busquet et al. 2013).

The filaments exhibit a nearly universal width of $\sim 0.1 \mathrm{pc}$ (measured by FWHM of the column density), independent of the central column density or length of the filament (Arzoumanian et al. 2011; Palmeirim et al. 2013). Furthermore, the radial dependence of the column density profile is flatter than expected for an isothermal cylindrical filament in hydrostatic equilibrium (Ostriker 1964). A number of explanations have been suggested for the observed density profiles: the filaments correspond to stagnant gas in locally colliding flows (Peretto et al. 2012), they are isothermal equilibrium cylinders in pressure-balance with the external medium (i.e., a radially-truncated cylinder in hydrostatic equilibrium; Fischera \& Martin 2012; Heitsch 2013), or are supported by magnetic fields (Fiege \& Pudritz 2000).

Thus, the origin of the filamentary cloud structure still remains unclear and heavily debated. The clouds can be formed by compressive motions of a gravitational and/or turbulent nature (Ballesteros-Paredes et al. 2007). Indeed, many numerical simulations produce filamentary structures by either isothermal-driven turbulence (e.g., Padoan \& Nordlund 2002; de Avillez \& Breitschwerdt 2005; Ballesteros-Paredes \& Mac Low 2002), thermal instability in large-scale convergent flows (e.g., Vázquez-Semadeni et al. 2003; Audit \& Hennebelle 2005; Heitsch et al. 2008) or behind a shock front (e.g., Koyama \& Inutsuka 2000; van Loo et al. 2007, 2010), or by gravitational instabilities in self-gravitating sheets (e.g., Nakajima \& Hanawa 1996; Umekawa et al. 1999).

Some of the observational evidence points to a turbulent formation process for filaments and dense cores in the ISM. However, observations often show cores within clouds with linewidths that require little additional support beyond thermal pressure (e.g., Myers 1983; Keto et al. 2004). These quiescent cores are adequately modeled by Bonnor-Ebert spheres, confining the cores by self-gravity and external pressure (Bonnor 1956; Alves et al. 2001; Tafalla et al. 2004). Such objects show that gravity plays a more prominent role than assumed in the turbulent models.

Magnetic fields are also important in the formation of the filaments. Studies comparing the orientation of the magnetic field with respect to the diffuse gas provide more insight (e.g., Li et al. 2013). Optical polarization measurements of the Taurus molecular cloud show that the magnetic field in the diffuse gas is orientated perpendicular to the axis of the B216 and B217 filaments (Goodman et al. 1992). This suggests that the filaments form as gas streams along the magnetic field lines (e.g., Ballesteros-Paredes et al. 1999). However, the long axis of the L1506 filament in Taurus and of the $\rho$ Ophiuchus clouds lies along the magnetic field (Goldsmith et al. 2008; Goodman et al. 1990).

In this paper, we focus on the role of self-gravity and magnetic fields on the formation of filaments and cores within selfgravitating layers. We neglect the effect of turbulent motions (other than those produced by gravitational and magnetic 
instabilities). Self-gravitating isothermal layers are unstable to perturbations if the exciting modes are of sufficiently long wavelengths (Ledoux 1951) and they fragment into clumps or thin filaments (Miyama et al. 1987). When the layers are threaded by magnetic fields, fragmentation still occurs. Using a linear perturbation analysis, Nagai et al. (1998) show that, for a layer with a thickness larger than the pressure scale height, the magnetic field suppresses the growth of perturbations perpendicular to the field. Perturbations along the field are unaffected so that parallel filaments form within the slab. On the other hand, for a layer with a thickness smaller than the scale height, filaments form with their axis orientated along the magnetic field. The separation of the filaments is given by the wavelength of the fastest growing mode. The filaments then condense into clumps and dense cores as long as the filaments are in a quasi-static equilibrium (Inutsuka \& Miyama 1992).

We extend the study of Nagai et al. (1998) into the non-linear regime and discuss the resulting structures. In Section 2, we describe the initial conditions of the self-gravitating layer and the numerical model used. Then, we discuss the fragmentation of the layer in the absence (Section 3) and presence of a parallel or perpendicular magnetic field (Sections 4 and 5). In Section 6, we interpret the structure of the molecular cloud G14.225-0.506 in terms of the discussed gravitational instability model. Finally, we conclude and discuss our results in Section 7.

\section{MODEL SET-UP}

\subsection{Initial Conditions}

For our initial conditions, we assume a self-gravitating isothermal layer that extends to infinity along the $x, y$ plane. The layer is initially in hydrostatic equilibrium, so that the density distribution along the $z$ axis is given by (Spitzer 1942)

$$
\rho(z)=\rho_{0} \operatorname{sech}^{2}(z / H),
$$

with $H=a / \sqrt{2 \pi G \rho_{0}}$ as the pressure scale height, $a$ as the isothermal sound speed, $G$ as the gravitational constant, and $\rho_{0}$ as the unperturbed density at the midplane (i.e., at $z=0$ ). The layer is truncated at $\pm z_{B}$ and confined by a constant external thermal gas pressure, $p_{\mathrm{g} \text {,ext }}=a^{2} \rho\left(z_{B}\right)$. In this paper, we will not study the effect of the external pressure on fragmentation. Therefore, we only consider a layer bounded by a low external pressure, i.e., $p_{\mathrm{g}, \mathrm{ext}} \ll p_{\mathrm{g}, 0}$. We adopt $z_{B}=2 H$, so that $p_{\text {ext }}=0.071 p_{\mathrm{g}, 0}$.

Outside the layer, not only the pressure, but also the density, $\rho_{\text {ext }}$, is kept constant at a small fraction of the density at the boundary (see Section 2.2 for the exact value). This means that the external temperature differs from the internal temperature. We, therefore, modify the isothermal equation of state to be

$$
p_{\mathrm{g}}=\rho\left((1-\alpha) \frac{p_{\mathrm{ext}}}{\rho_{\mathrm{ext}}}+\alpha a^{2}\right) .
$$

This reflects the difference in external and internal temperature with $\alpha$ as a scalar that is one inside the layer and zero outside.

The layer and the external medium are threaded by a uniform magnetic field. For parallel models, the magnetic field is along the $x$ axis, while, for perpendicular models, it is along the $z$ axis. The field strength is given by $\beta=p_{m} / p_{g}$ at the midplane with $p_{m}=B^{2} / 2$ as the magnetic pressure. For our models, we use $\beta=\infty$ (i.e., no magnetic field), 10, 1, and 0.1 . For simplicity, our simulations are performed dimensionless. We adopt $\rho_{0}=2 \pi, G=1$ and $a=1$ so that $H=1 / 2 \pi$ and the dynamical time, $t_{\mathrm{dyn}}=1 / \pi$. The free-fall time associated with gas at the midplane is $\sim 1 / 5$. For a layer with a central density of $1000 \mathrm{~cm}^{-3}$ (and thus a surface density of $\approx 10^{21} \mathrm{~cm}^{-2}$ ) and a temperature of $10 \mathrm{~K}$, these values correspond to $H=0.159 \mathrm{pc}$, $B=27 \mu \mathrm{G}$ (for $\beta=1.0$ ) and $t_{\mathrm{dyn}}=1.57 \mathrm{Myr}$. In the rest of the paper, we will evaluate the dimensionless results in terms of this layer.

This set-up is similar to the one used in the linear analysis of Nagai et al. (1998), allowing for a comparison between the analytically and numerically derived growth rates of the gravitational instability. To study the gravitational instability of a pressure-confined self-gravitating layer, we perturb the equilibrium state. We do this in two different ways, i.e., we superimpose the density either with a sinusoidal density perturbation of a given wavelength or with randomly generated noise. The former is used to determine the growth rates to compare with analytic values, while the latter shows how different wave modes interact with each other. From the linear analysis Nagai et al. (1998) suggest that first the filaments form and only then the dense cores.

\subsection{Numerical Code and Domain}

To solve the ideal magnetohydrodynamics (MHD) equations, we use the Adaptive Mesh Refinement (AMR) MHD code MG (Van Loo et al. 2006; Falle et al. 2012). The basic algorithm is a second-order Godunov scheme with a linear Riemann solver (Falle 1991). To ensure that the solenoidal constraint is met, a divergence cleaning algorithm is implemented in the numerical scheme (Dedner et al. 2002). A hierarchy of grid levels are used with a mesh spacing on grid level $n$ of $\Delta x / 2^{n}$ with $\Delta x$ the cell size at the coarsest level. While the two coarsest levels cover the entire domain, finer levels generally do not. Refinement is on a cell-by-cell basis and is controlled by error estimates based on the difference between solutions on different grid levels. Self-gravity is computed using a full approximation multigrid to solve the Poisson equation.

The numerical domain is given by $-2<z<2$ and $-L / 2<$ $x, y<L / 2$ where $L$ is either $\lambda_{\text {crit }} / 2, \lambda_{\text {crit }}, \lambda_{\text {max }}$, or $4 \lambda_{\text {max }}$. Here, $\lambda_{\text {crit }}=2 \pi H$ is the critical wavelength below which the gravitational instability is suppressed and $\lambda_{\max }=4 \pi H$ is the wavelength for which the growth rate is maximal (see Nagai et al. 1998). For our adopted values, $\lambda_{\text {crit }} \approx 1$ and $\lambda_{\max } \approx 2$. As the layer is assumed to be infinite, we set the boundary condition on both the $x$ and $y$ axis to periodic. For the $z$ axis, we use free-flow boundary conditions.

The resolution of the simulation is set by the vertical extent of the slab. It is necessary to properly resolve the pressure scale height, $H$, in order to find a proper balance between pressure gradients and self-gravity. We resolve the scale height by at least five cells. Therefore, we use an AMR mesh with the coarse level having four grid cells along the axis with the shortest length (along the $x, y$ axis for the runs up to $L=\lambda_{\max }$ and along the $z$ axis for $L=4 \lambda_{\max }$ ) and 128 cells along the $z$ axis for the finest grid level. This means that the number of additional grid levels varies with different models, i.e., from two extra grid levels for $L=\lambda_{\text {crit }} / 2$ up to five grid levels for $L=4 \lambda_{\max }$. This resolution is more than sufficient to resolve the Jeans' length as suggested by Truelove et al. (1997) to avoid artificial fragmentation. In fact, artificial fragmentation is only expected above $\rho>\pi a^{2} / 16 G \Delta x^{2} \approx 200$.

Because we are interested in the gravitational instability in the equilibrium layer, we need to ensure that the external medium 


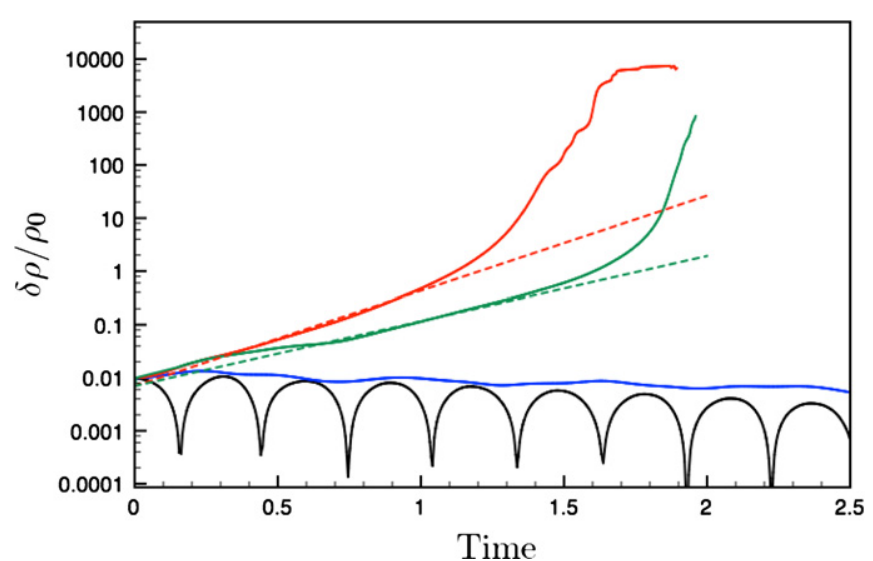

Figure 1. Temporal evolution of the normalized density perturbation amplitude for a wave mode with a wavelength of $\lambda_{\text {crit }} / 2$ (black), $\lambda_{\text {crit }}$ (blue), $\lambda_{\max }$ (red), and $4 \lambda_{\max }$ (green). The dashed lines show the linear evolution using the theoretically derived growth rates. For a layer with a central density of $1000 \mathrm{~cm}^{-3}$, i.e., our default layer, a unit of time is $\sim 4.9 \mathrm{Myr}$.

(A color version of this figure is available in the online journal.)

does not affect the gravitational instability in the layer in any way. By assuming a constant pressure in the external medium, the external medium needs to be a vacuum. (The gravitational force is zero if there is no pressure force.) However, in a grid code, we cannot set the density to zero. For a non-zero external density, the self-gravity of the layer drags the external medium toward it. Then the external medium affects the hydrostatic layer because gas is accreted onto the layer and because the ram pressure contributes to the total external pressure. We find that the external density needs to be $10^{-4} \rho\left(z_{\mathrm{B}}\right)$ at most not to affect the equilibrium layer. As the external pressure is $\rho\left(z_{\mathrm{B}}\right) a^{2}$, this means that the external sound speed is $10^{2}$ times larger than in the layer. This significantly limits the stable numerical time step derived from the Courant condition, $\approx \Delta x / a$. Due to this constraint, we use a different approach, i.e., we artificially switch off the self-gravity in the external medium. This is easily done because the different media have a different value of the scalar $\alpha$. This intervention allows us to set the external medium density to $0.1 \rho\left(z_{\mathrm{B}}\right)$ without affecting the equilibrium layer and gives us a speed up of more than a factor of ten in computational time.

\section{NO MAGNETIC FIELD}

In order to determine the effect of the magnetic field on the density distribution and geometry of the filaments, we first study the onset and evolution of the gravitational instability in the absence of a magnetic field. We start by confirming the theoretically derived growth rates and continue by examining the interaction of different wave modes.

Growth rates. Figure 1 shows the evolution of the maximum density perturbation amplitude for modes with a wavelength of $\lambda_{\text {crit }} / 2, \lambda_{\text {crit }}, \lambda_{\max }$, and $4 \lambda_{\max }$. The initial amplitude of the perturbation is $1 \%$ of the local density. For a perturbation with a wavelength below $\lambda_{\text {crit }}$, the amplitude of the perturbation gradually decreases. The wave mode is damped and the selfgravitating layer is stable under such a perturbation. This remains so until the wavelength of the perturbing mode reaches $\lambda_{\text {crit }}$. This critical wavelength (hence $\lambda_{\text {crit }}$ ) has a zero growth rate and the amplitude of the critical wave mode remains roughly constant for multiple dynamical time scales. It also marks the transition to a layer that is unstable to perturbations with $\lambda>\lambda_{\text {crit }}$. Our simulations reproduce the theoretically derived

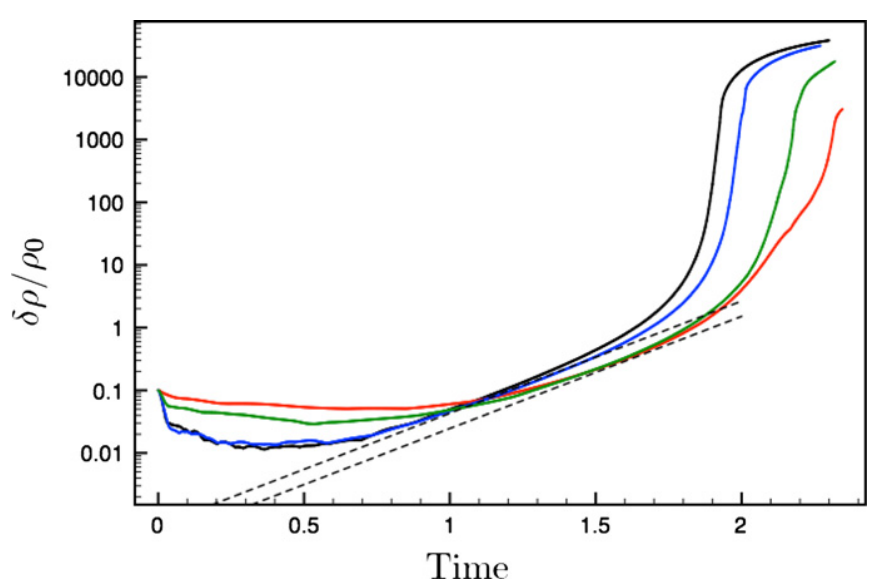

Figure 2. Temporal evolution of the normalized density perturbation (solid line) when random density perturbations are imposed on the equilibrium slab for a hydrodynamical (black), $\beta=10$ (blue), 1 (green), and 0.1 (red) model. The dashed lines show the linear growth of a wave mode with a wavelength, $\lambda_{\max }$. For our default layer, the unit of time is $\sim 4.9 \mathrm{Myr}$.

(A color version of this figure is available in the online journal.)

linear growth rates of Nagai et al. (1998) for these longer wavelength perturbations very well. During the linear growth phase, the amplitude of the perturbation grows as $\sim \exp (\omega t)$. For the fastest growing wave mode with wavelength $\lambda_{\max }$, we find $\omega \approx 4.12$, which is close to the analytically derived value of $\omega \approx 4.49$ (Elmegreen \& Elmegreen 1978). For $4 \lambda_{\max }, \omega \approx 2.81$ and is similar to the value inferred from Figure 1 of Nagai et al. (1998). Note that the linear growth phase ends when $\delta \rho \approx \rho_{0}$ and the growth becomes non-linear. This non-linear growth phase lasts until nearly all the gas of the layer is concentrated in a single dense filament which is starting to fragment into cores. We do not have sufficient resolution to follow this fragmentation and, at this time, we end the simulation.

Filament and core formation. We have confirmed the growth rates for different wave modes, but we need to understand how the different wave modes interact with each other. Therefore, we superimpose the equilibrium density with random noise. The maximal amplitude of the density noise is $10 \%$ of the local density. We allow wave modes with a wavelength up to $4 \lambda_{\max }$ to develop within the layer, i.e., the numerical domain is $-2 \lambda_{\max }<$ $x, y<2 \lambda_{\max }$. Figure 2 shows the maximum amplitude of the density perturbations. Initially, as we impose random noise at the grid level, the wave modes have a wavelength below $\lambda_{\text {crit }}$. These modes are stable and the amplitude thus decreases. However, wave modes with longer wavelengths for which the layer is unstable are excited and gradually grow. The fastest growing unstable wave mode, i.e., the mode with a wavelength $\lambda_{\max }$, becomes dominant. This can be clearly seen in Figure 2 as the evolution of the maximum perturbation amplitude can be described by the linear growth phase of that wave mode.

Figure 3 shows the resulting surface density integrated along the $z$ axis at two different times, i.e., at the beginning of the linear growth phase at $t=1$ and near the end of the non-linear growth phase at $t=2$. A network of interconnecting filaments with embedded cores has formed. Note that regions of high column density are already present early on in the linear phase. In fact, there is a one-to-one correlation. To better understand the interplay between the different filaments and the positions of the cores, we use a ridge finding algorithm to expose the skeleton of the network and to locate column density maxima (e.g., Lindeberg 1998). The ridges and local maxima for the right 

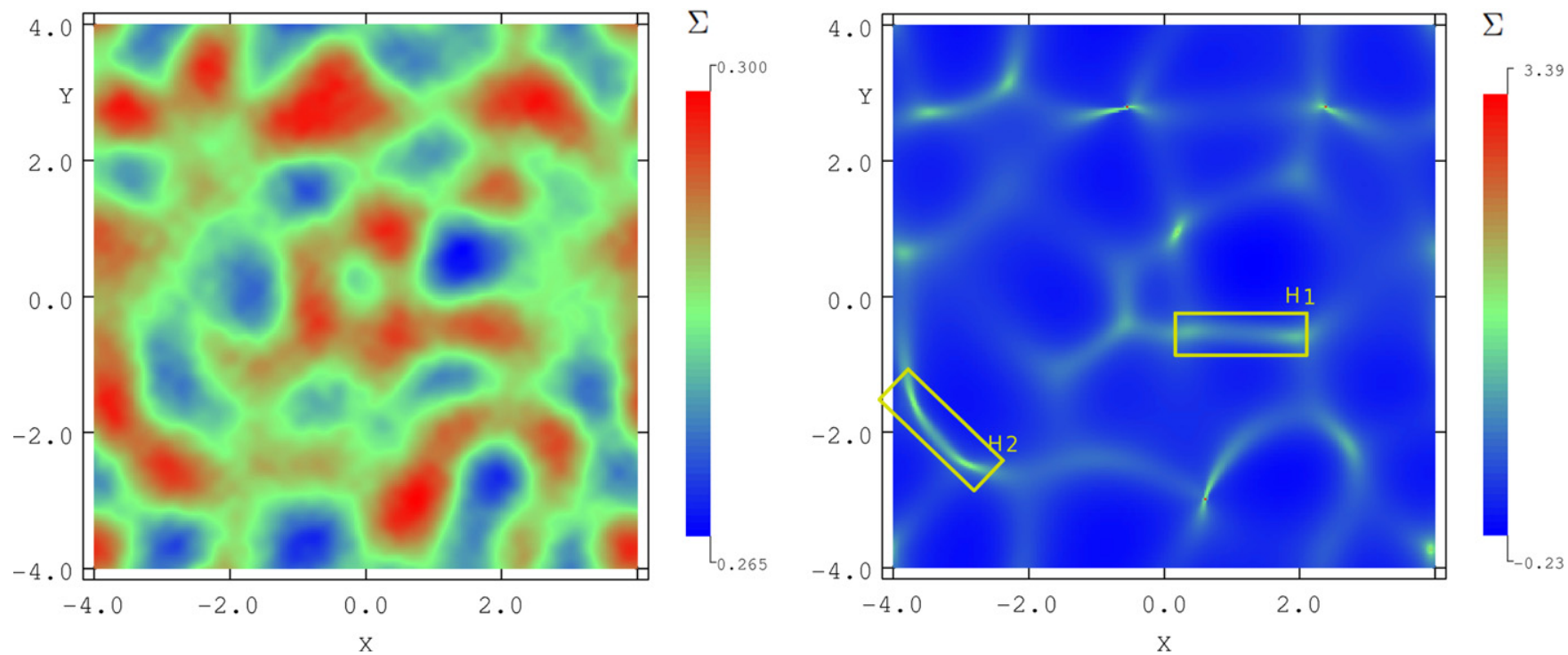

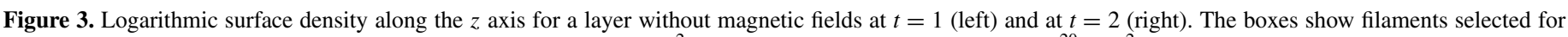
further analysis. In terms of our default layer, the area shown is $8 \times 8 \mathrm{pc}^{2}$ and the unit of surface density is $5 \times 10^{20} \mathrm{~cm}^{-2}$.

(An animation and color version of this figure are available in the online journal.)

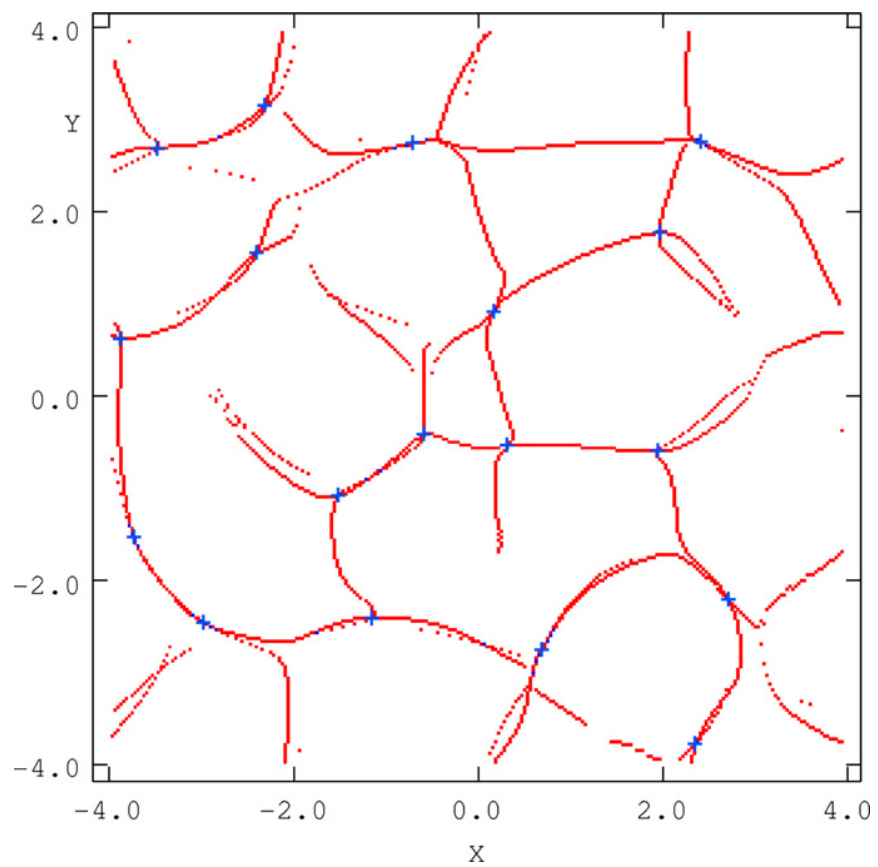

Figure 4. Ridge identification for the column density of the right panel in Figure 3. The local maxima are indicated by blue crosses.

(A color version of this figure is available in the online journal.)

panel of Figure 3 are shown in Figure 4. Note that filaments have a separation of about $\lambda_{\max }$ indicating that the filaments are indeed formed by the most unstable wavelength (as already inferred from the growth rates). Although, in the absence of magnetic fields, the most unstable wave mode has the same growth rate in all directions, filaments predominantly form along the $x$ and $y$ axes. This is most likely because the flow equations are evolved along the Cartesian coordinate axes in the numerical code and thus excite the wave modes along these directions. Another interesting feature is that the local maxima, i.e., dense cores, lie at the intersections of the filaments. This is reminiscent of the hub-filament structures discussed by Myers (2009) and the network of filaments seen in the Herschel observations of the Rosette molecular cloud and Pipe nebula (Schneider et al. 2012; Peretto et al. 2012). Such a network is also present in cloud formation simulations which include turbulence (e.g., VázquezSemadeni et al. 2003), but is generated due to dynamical (other than gravity) and thermal instabilities. Other random noise initializations produce a similar network of filaments with a separation of about $\lambda_{\max }$ and dense cores at the intersections of filaments.

Radial profile of filaments. Several authors (e.g., Arzoumanian et al. 2011; Palmeirim et al. 2013; Kirk et al. 2013) characterize the filaments by mapping the observed column densities with Plummer-like profiles. They assume that the underlying density profile of the cylindrical filament is given by

$$
\rho(r)=\frac{\rho_{0}}{\left[1+\left(r / R_{0}\right)^{2}\right]^{\frac{p}{2}}},
$$

where $r$ is the cylindrical radius, $R_{0}$ is the characteristic radius of the flat inner region, and $p$ is the power-law index of the profile at large radii. For $p=4$, this profile describes an isothermal cylinder in hydrostatic equilibrium with $R_{0}=$ $\sqrt{2 a^{2} / \pi G \rho_{0}}$ the scale height (Ostriker 1964). The resulting column density profile (assuming that the filament lies within the plane perpendicular to the integration) is also a Plummer-like profile given by

$$
\Sigma(l)=\frac{B_{p} \rho_{0} R_{0}}{\left[1+\left(l / R_{0}\right)^{2}\right]^{\frac{p-1}{2}}},
$$

where $B_{p}$ is the Euler beta function with input values $1 / 2$ and $(p-1) / 2$ and $l$ the projected radius.

We extract several filaments from the column density map at $t=2$. For each filament, we derive the radial distributions perpendicular to the axis at each cell along the filament axis. By combining all the distribution along a filament axis we obtain the average radial distribution of the filament. Note that, for curved filaments, radial profiles will cross each other, but this will not strongly influence the average radial distribution as long as the curvature is small. We then fit the average distribution 

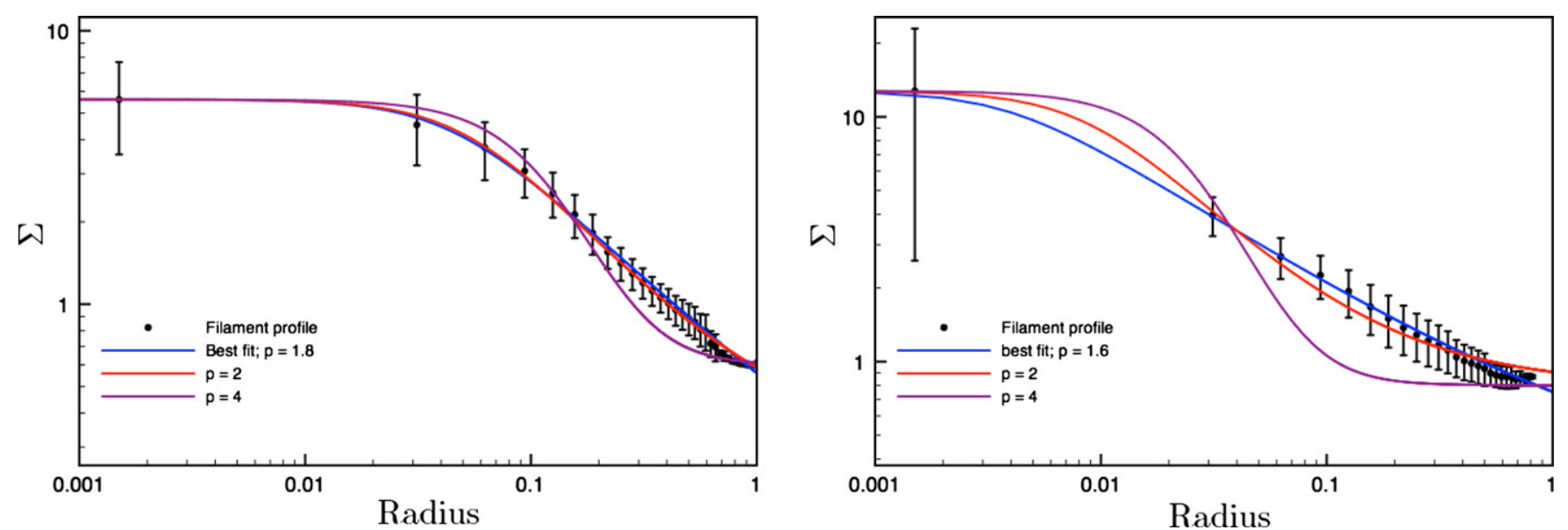

Figure 5. Radial column density profile for two selected regions in Figure 3: Region $\mathrm{H} 1$ is in the left panel, while Region $\mathrm{H} 2$ is in the right one. We display the central column density at a radius of 0.0015 and not at a radius of 0.0 . The solid circles show the column densities at a given radius from the filament axis averaged over the entire filament, while the error bars are the dispersion on the mean. The blue line is the best-fit Plummer profile with a background column density, while the red and purple lines show the Plummer-like profile for $p=2$ and $p=4$, respectively. For our default layer, the unit of length is $1 \mathrm{pc}$ and the unit of surface density is $5 \times 10^{20} \mathrm{~cm}^{-2}$.

(A color version of this figure is available in the online journal.)

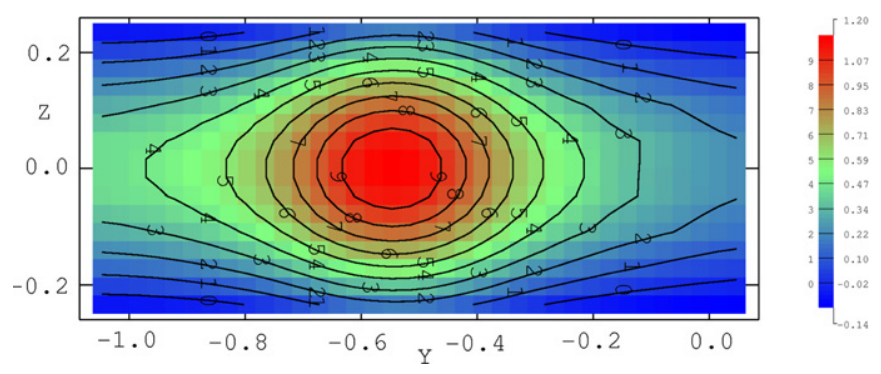

Figure 6. Density cross-section of the filament in Region H1 of Figure 3 at $x=1.25$ with contour lines. For the default layer, the units of density and length are $159.2 \mathrm{~cm}^{-3}$ and $1 \mathrm{pc}$, respectively.

(A color version of this figure is available in the online journal.)

with the theoretical profiles of Equation (4). We add a constant background column density because the filaments are embedded in a layer.

Figure 5 shows the profiles and fits for two filaments. The best fit for Region $\mathrm{H} 1$ is given by $p=1.8, R_{0}=0.045$, and $\rho_{0}=26.9$, while $p=2$ still produces a good fit. However, an equilibrium model with $p=4$ cannot reproduce the filament profile at all. A similar result is found for Region $\mathrm{H} 2$ where the best fit is $p=1.6, R_{0}=0.004$, and $\rho_{0}=683$. These results agree well with observationally derived values of $1.5<p<2.5$ by Arzoumanian et al. (2011) and others. The value of $R_{0}$, however, varies significantly between the different filaments. For Region $\mathrm{H} 1$ the inner flat region is about an order of magnitude larger than in Region $\mathrm{H} 2$.

The average radial column density profiles suggest that the filaments are far from equilibrium. Numerical simulations provide the opportunity to confirm this claim directly from the density distribution. We examine different slices across the filament in Region H1. Figure 6 shows one of these crosssections. Although the center of the filament is nearly axisymmetric, the outer layers are flattened along the slab. An equilibrium profile (Ostriker 1964) provides a good fit to the central region of the filament, but cannot explain the asymmetry along the different axes (see Figure 7). This, however, does not imply that the structure is far from equilibrium. Actually, the cross-section is reminiscent of a modulated self- gravitating layer in equilibrium (Curry 2000; Myers 2009). The density distribution of such a modulated layer is given by (Schmid-Burgk 1967)

$$
\rho=\rho_{c} \frac{1-A^{2}}{(\cosh (z / L)-A \cos (x / L))^{2}},
$$

where $\rho_{c}$ is the midplane density of the unmodulated layer, $A$ is the modulation factor between zero and one, and $L=$ $a / \sqrt{2 \pi G \rho_{c}}$ is the scale height. For $A=0$, we find the Spitzer solution for an infinite layer, i.e., Equation (1), while, for $A \rightarrow 1$, it converges to the Ostriker (1964) solution for an infinite cylinder. By applying a Schmid-Burgk profile to density slices through the center of the cross-section shown in Figure 6 and along the $y$ and $z$ coordinate axes, ${ }^{1}$ we find that the density profile of the filament is best reproduced with $A=0.6$ and $\rho_{c}=3.976$ (see Figure 7). The fit is good for radii up to the scale height $L=0.2$, above which the density along the midplane is higher. Other slices of the filament can also be described by a Schmid-Burgk profile albeit with somewhat different values of $A$ and $\rho_{c}$ (i.e., $0.55<A<0.65$ and $4<\rho_{c}<7.5$ ). This variation implies a density variation along the filament axis and reflects that cores are embedded within a filament.

By integrating Equation (5) along the $z$ axis and using the derived parameters, $A$ and $\rho_{c}$, we find that the Schmid-Burgk profile reproduces not only the density profile, but also the column density profile (see Figure 7). Again the fit is best for radii below the scale height, but it exhibits a flatter radial distribution than the (Ostriker 1964) profile. This partly explains the equilibrium radial profile flatter than the Ostriker observed in the mean column density distribution (Figure 5). Some of the flattening is due to averaging profiles with marginally different parameters.

Evolution of the filaments. Although the density profile shows a filament close to equilibrium, it is in fact dynamically evolving. The filament profile changes with time, but can, at all instances, be described by a Schmid-Burgk profile (see Figure 8). Initially, the filament is only a small perturbation on top of the equilibrium

\footnotetext{
1 The slice along the $z$ axis is not at $z=0$, but at $z=\Delta x / 2$ due to the gridding of the numerical domain.
} 

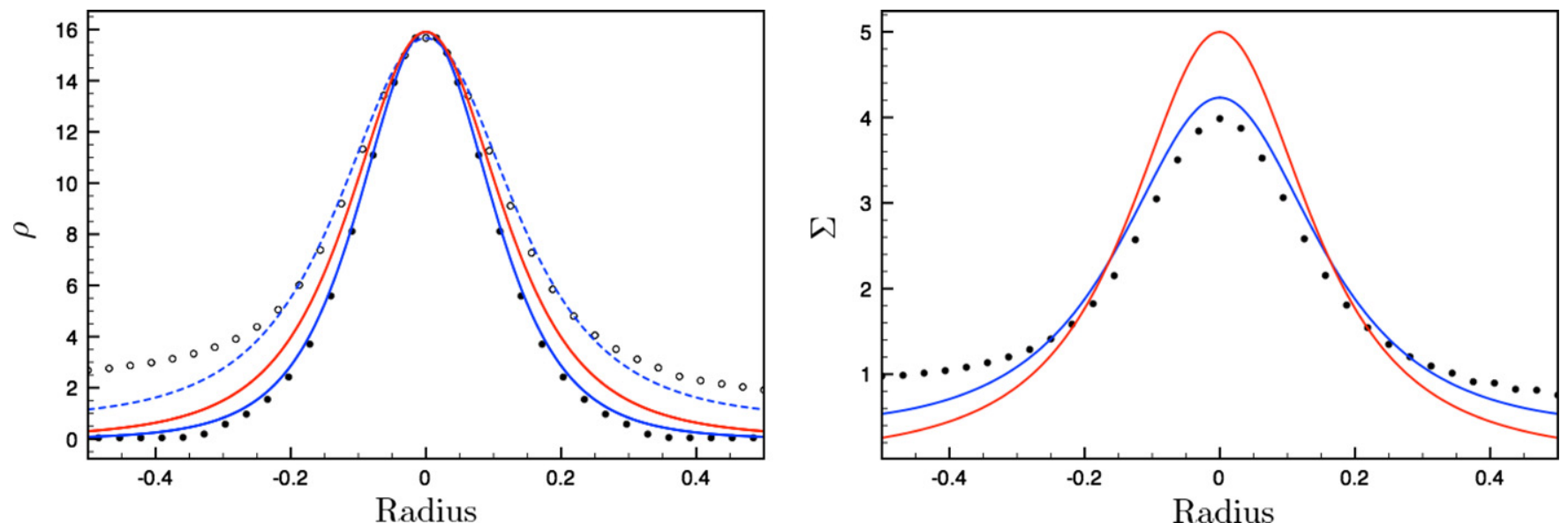

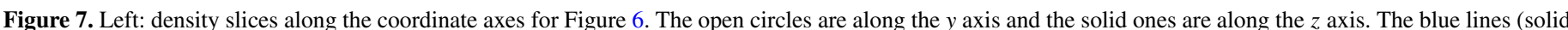

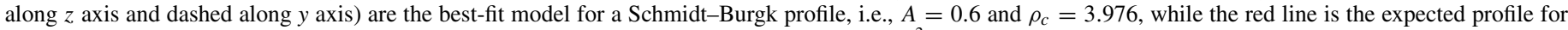

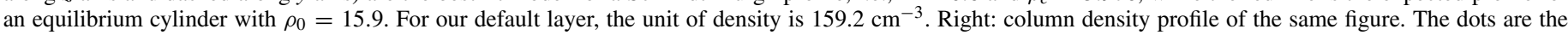

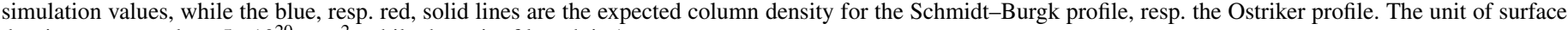
density corresponds to $5 \times 10^{20} \mathrm{~cm}^{-2}$, while the unit of length is $1 \mathrm{pc}$.

(A color version of this figure is available in the online journal.)

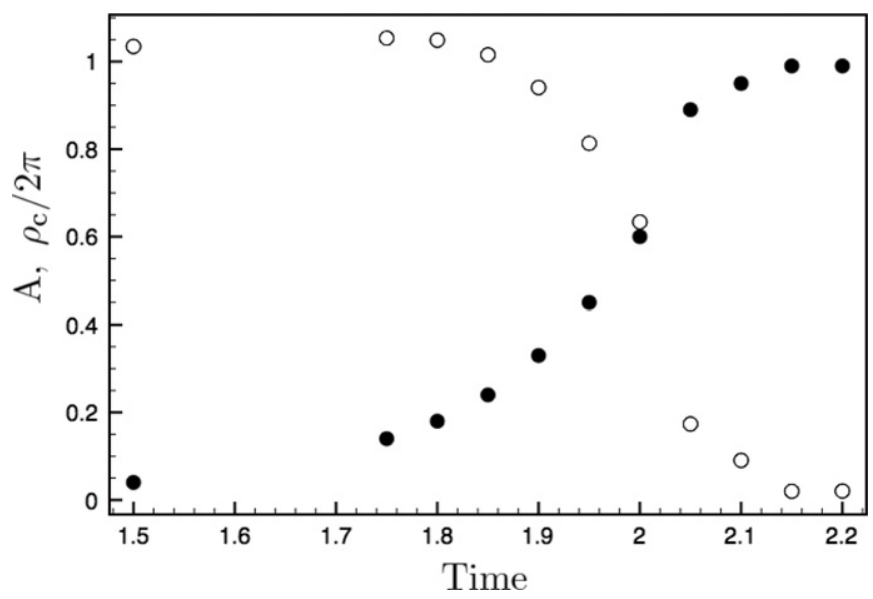

Figure 8. Evolution of the filament profile as a function of time. The solid circles are the values for $A$, while the open ones are the values of $\rho_{c}$, normalized to the initial value. In terms of our default layer, a unit of time is $4.9 \mathrm{Myr}$.

layer (i.e., $A \approx 0$ ), while it evolves toward an equilibrium cylinder at the end of the non-linear phase (i.e., $A \approx 1$ ). The evolution of a filament thus occurs through a sequence of quasiequilibrium distributions. Note that $\rho_{c}$ does not remain constant during the evolution. This suggests that gas does not accumulate in this filament segment. Figure 9 indeed shows that, while gas flows toward the filaments, it is then diverted toward denser regions (cores) along the filament axis.

The evolution of the filaments is set by the mass that the gravitational instability sweeps up. The expected line-mass is essentially determined by the wavelength of the fastest growing wavelength and given by $m_{\text {line }} \approx \Sigma_{0} \lambda_{\max }$ with $\Sigma_{0} \approx 2 \rho_{0} H$ the column density of the equilibrium layer. This value needs to be compared with the critical value for the line-mass of a stable cylinder, i.e., $m_{\text {crit }}=2 a^{2} / G$ (Ostriker 1964). For our model parameters, $m_{\text {line }} \approx 1.9 m_{\text {crit }}$. We can also derive linemasses for different cross-sections in filaments. For example, we find $m_{\text {line }} \approx 2.6$ for Figure 7 ). While this is less than the predicted value, it still exceeds the critical value of $m_{\text {crit }}=2$. It is interesting to note that half of the line-mass lies within the central region bound by the scale height. Thus, the filament is

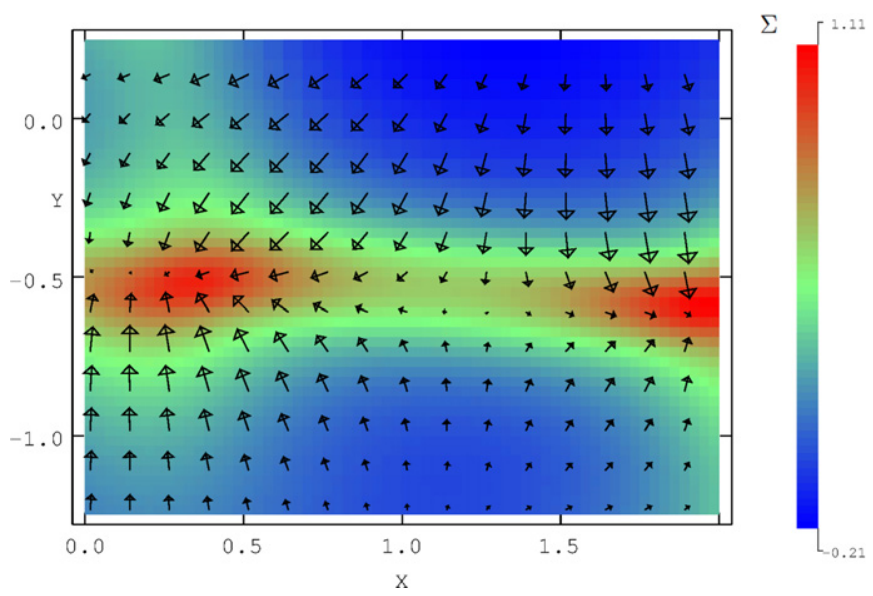

Figure 9. Logarithmic column density for Region $\mathrm{H} 1$ of Figure 3. The vectors represent the mass-weighted velocities in the plane. Their size is an indication of their magnitude with the maximal velocity shown 1.6 times the sound speed. Remember that the unit of column density is $5 \times 10^{20} \mathrm{~cm}^{-2}$ and that the sound speed is $\approx 0.2 \mathrm{~km} \mathrm{~s}^{-1}$ in our default layer.

(A color version of this figure is available in the online journal.)

collapsing radially while maintaining a quasi-equilibrium for radii up to the scale height.

Evolution of the dense cores. As mentioned earlier, the dense cores arise at the junctions of the filaments. The cores are elongated along the axis of the most dense filament. As they evolve, the cores become more centrally condensed (see Figure 10).

The cores collapse at a rate that is slower than expected for pressureless gravitational, i.e., homologous, collapse of a uniform sphere (the free-fall time is between 0.1 and 0.2 for all the cores). There are two main reasons. Firstly, the cores are embedded in and are formed out of cylindrical filaments. Mass accretion toward the cores is thus not isotropic but highly directional. Toalá et al. (2012) and Pon et al. (2012) show that the collapse time scales of non-spherical structures are longer than the corresponding spherical free-fall time scale. In the case of a cylindrical cloud, the time-scale depends strongly on the aspect ratio of the cloud. 


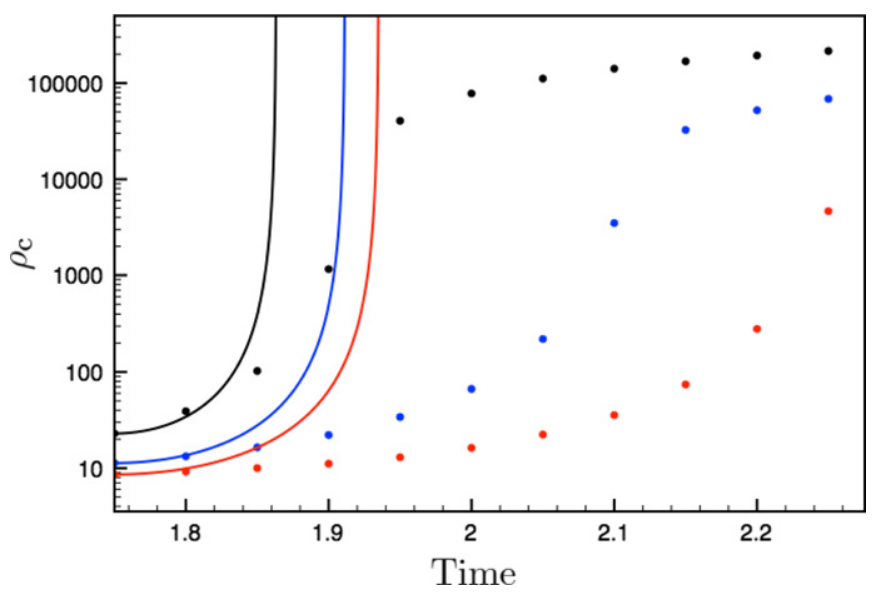

Figure 10. Evolution of the central density of three selected cores in the hydrodynamical simulation (full circles). Each core is represented by a different color (black, blue, or red). The solid lines show the evolution of the central density for homologous collapse of a uniform sphere for each core (in the same color). In terms of the default layer, a unit of time equals 4.9 Myr and a unit of density $159.2 \mathrm{~cm}^{-3}$.

(A color version of this figure is available in the online journal.)

Note that the above authors only consider the collapse of constant mass structures. The mass of the filaments does change in our simulation. However, as the line-mass of the filament is set by the fastest-growing wavelength, the mass of the filaments does not change significantly once the evolution is in the nonlinear regime.

Secondly, the assumption of pressureless collapse is not valid. The results for the filaments show that the inner region can be adequately described by an equilibrium profile in which selfgravity is nearly balanced by thermal pressure. Inutsuka \& Miyama (1992) show that, in the case of equilibrium cylinders, this significantly reduces the collapse rate. As cores form out of filaments, it is likely that the thermal pressure plays a similar role in cores. However, we cannot easily test this hypothesis as there is no analytic solution for an equilibrium core within a cylindrical filament (and certainly not for a core in a Schmid-Burgk-like filament). Furthermore, the accretion of gas is not along one filament, but multiple ones, complicating the expected structure even more.

\section{PARALLEL MAGNETIC FIELD}

Growth rates. The analysis of Nagai et al. (1998) shows that the gravitational instability in an equilibrium layer is modified by magnetic fields as they stabilize instabilities propagating perpendicular to the field (when the external pressure is small). Instabilities along the magnetic field are not affected. The effect of the magnetic field is expressed as a function of a dimensionless parameter, $\alpha^{\prime}$, which for our model parameters is given by $\alpha^{\prime}=2.15 / \beta$. Thus, our models examine a range of $\alpha^{\prime}$ between 0.215 and 21.5. For values as low as $\alpha^{\prime}=1.25$ (i.e., $\beta=2$ ) the perpendicular perturbations are already strongly suppressed (see, e.g., Figure 4 of Nagai et al. (1998)).

Similar to the hydrodynamical simulations, we first examine whether our numerical model reproduces the expected linear growth rates. We only test this for $\beta=0.10$ (i.e., $\alpha^{\prime}=21.5$ ). For perturbations along the magnetic field (i.e., along the $x$ axis), we find, as expected, linear growth rates that are marginally smaller than in the hydrodynamical model (see Figure 11). The critical wavelength and the maximal wavelength are also the same as before. For perpendicular perturbations along the $y$ axis,

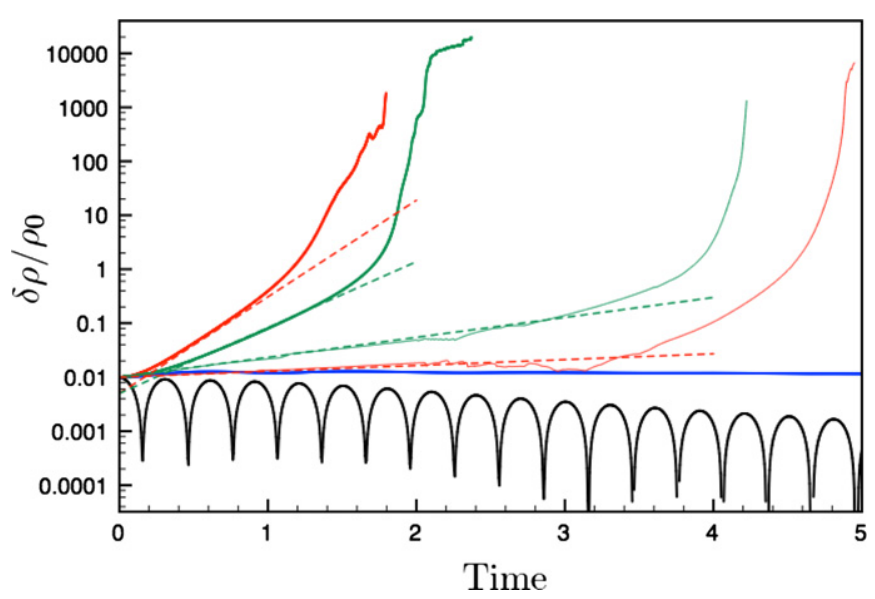

Figure 11. Temporal evolution of the normalized density perturbation for perturbations with a wavelength of $\lambda_{\text {crit }} / 2$ (black), $\lambda_{\text {crit }}$ (blue), $\lambda_{\max }$ (red), and $4 \lambda_{\max }$ (green). The thin solid lines are for perturbations along the $y$ axis. The dashed lines show the linear evolution using the theoretically derived growth rates. As before, the unit of time corresponds to 4.9 Myr for our default layer.

(A color version of this figure is available in the online journal.)

the growth rates are significantly reduced. For $\lambda_{\max }$ we find $\omega \approx 0.25$ or about 20 times lower than for an identical perturbation along the $x$ axis, while we have $\omega \approx 0.85$ when $4 \lambda_{\max }$. Note that the growth rate for $4 \lambda_{\max }$ is larger than for $\lambda_{\max }$ as $\lambda_{\max }$ is here the value for the fastest growing wave mode along the $x$ axis and not the $y$ axis. The critical and maximal wavelength along the $y$ axis are shifted toward longer wavelengths, i.e., $\lambda_{\mathrm{y}, \max } \approx 2.5 \lambda_{\max }$ and $\lambda_{\mathrm{y}, \text { crit }} \approx 1.8 \lambda_{\text {crit }}$. The shown models for perturbations perpendicular to the magnetic field then represent models close to the critical and maximal wavelength.

The simulations confirm the behavior derived from the linear analysis and show that the magnetic field introduces a preferred direction for the growth of perturbations, even for high values of $\beta(>1)$.

Filament formation. We superimpose the equilibrium layer with the same random noise perturbations as the hydrodynamical model. Figure 2 shows the normalized maximum perturbation amplitude for $\beta=10,1$, and 0.1 (as well as for $\beta=\infty$, i.e., the hydrodynamical model). The linear phase of the instability shows, for all of the magnetic models, the typical growth rate of a mode with a wavelength of $\lambda_{\max }$. That this specific wave mode is dominant also becomes apparent from the column density structure (see Figure 12). As the perpendicular perturbations are significantly damped for the $\beta=0.1$ model (see above), only the modes parallel to the magnetic field become unstable. Four parallel filaments are then produced with a separation of about $\lambda_{\max }$. Note that the $\beta=0.1$ model is shown at $t=2.25$ and not $t=2$ as it reaches similar densities at later times than in the $\beta=10$ model.

Although the layer is threaded by a magnetic field, the column density structure of the $\beta=10$ model shows filaments both parallel and perpendicular to the magnetic field direction. In fact, the column density structure is very similar to the structure seen in the hydrodynamical model. Actually, the positions of the filament ridges are identical in both models (see Figure 13). The ridge positions for the stronger magnetic field models (i.e., $\beta=1$ and 0.1 ) are also interchangeable. Remember that the density initialization is identical for all models and the subsequent evolution is then determined by the growth of the unstable modes. This means we can identify two different regimes, i.e., a quasi-hydrodynamical regime and a strong 

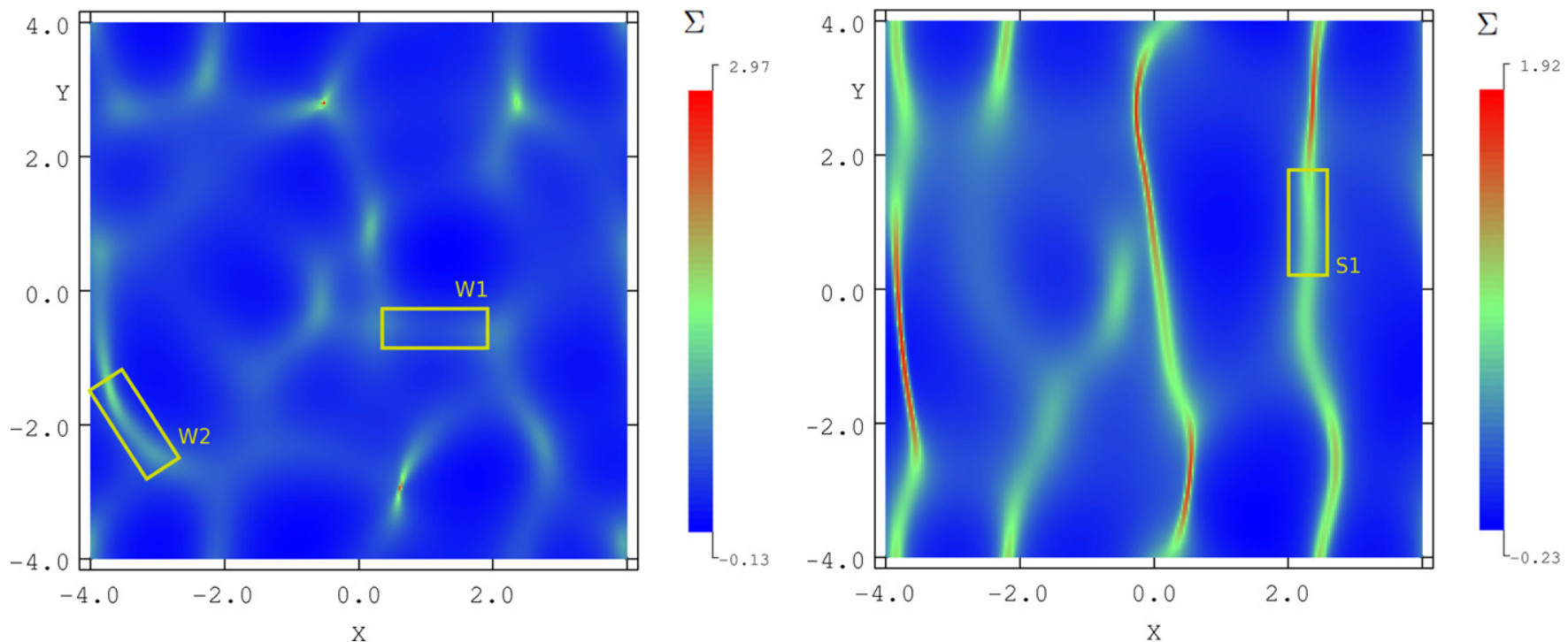

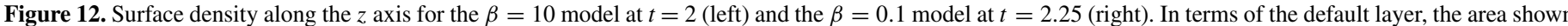
is $8 \times 8 \mathrm{pc}^{2}$ and the unit of surface density is $5 \times 10^{20} \mathrm{~cm}^{-2}$.

(Animations and color version of this figure are available in the online journal.)
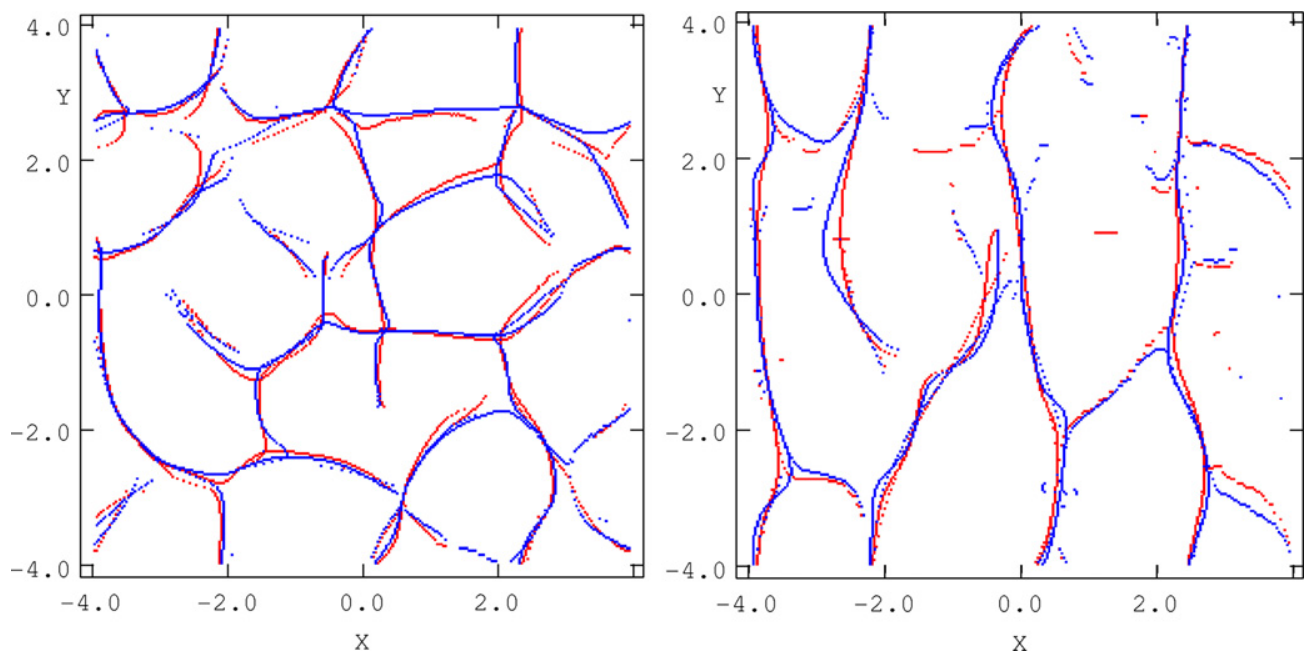

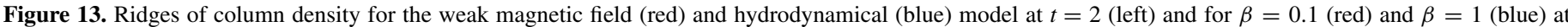
$t=2.25$ (right)

(A color version of this figure is available in the online journal.)

magnetic field one. The separation between the two states lies somewhere between $\beta=2$ and 10 .

Not only the density structure, but also the magnetic field structure is distinctively different in the two regimes. In the strong magnetic regime, the magnetic field is dynamically important and suppresses perturbations perpendicular to its orientation. Then gas flows are predominantly along the magnetic field lines resulting in filaments perpendicular to the magnetic field. On the other hand, in the weak magnetic regime, the magnetic field is dynamically passive and is dragged with the gas. Consequently, the density structures lie not only perpendicular to the field lines, but also parallel and oblique.

For the oblique filaments, an interesting phenomenon occurs: while the magnetic field is oblique to the filament axis in the outer regions of the filament, it is orientated along the filament axis within its central region (see Figure 14). The filaments are bound by fast-mode shocks in which the magnetic field increases perpendicular to the shock normal. The formation of such filaments is described in Nakajima \& Hanawa (1996) who show that such filaments are in quasi-static equilibrium in their inner parts.

Radial filament profile. The average radial column density profile for the filaments in the magnetized layers is similar to the profiles extracted for the hydrodynamical model (see Figures 15 and 16). The distribution is best fitted by Plummer profiles (Equation (3)) with power-law indices $p \leqslant 2$ and cannot be fitted by an equilibrium profile with $p=4$. An exception is Region $\mathrm{W} 1$ which can be fitted reasonably well by a large range of $p$ values, including $p=4$. The ratio of the central-to-external column density for Region W1 is small. The distribution then does not properly sample the power-law tail of the Plummer profile because the distribution is dominated by the flat inner region and the background column density. This results in a broad range of possible power-law indices for the Plummer profile.

Again, density slices through the filaments show that the density distribution of the central region can be approximated by an equilibrium Schmid-Burgk profile. For example, the density 


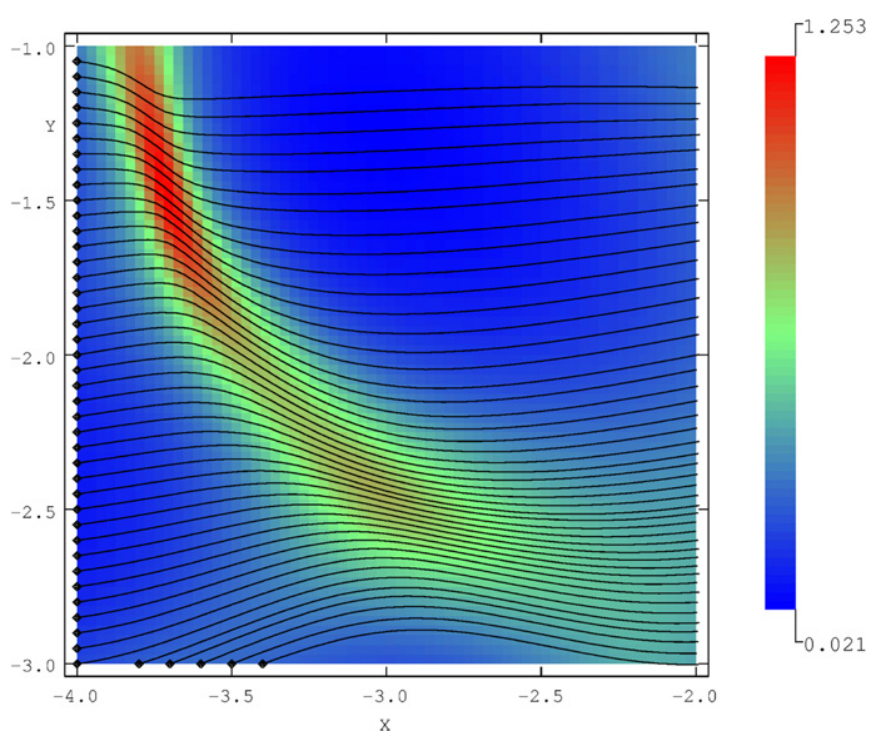

Figure 14. Column density of Region W1 with the vectors showing the massweighted magnetic field. Their length indicates the magnetic field magnitude with the maximum about three times the initial magnitude. In terms of the default layer, the unit of column density is $5 \times 10^{20} \mathrm{~cm}^{-2}$ and the maximum magnetic field strength displayed is $6 \mu \mathrm{G}$.

(A color version of this figure is available in the online journal.)

distribution for a slice at $x=1.25$ in Region $\mathrm{W} 1$ is best fitted with $A=0.13$ and $\rho_{c}=4.825$. A slice through $y=1$ of Region $\mathrm{S} 1$ is best fitted by an Ostriker cylinder (or a Schmid-Burgk profile with $A=1$ ) with $\rho_{c}=24.9$ (see Figure 17). The selection of these filaments represents different stages during the formation process, i.e., the filament of Region W1 is starting to form, while Region $\mathrm{S} 1$ shows the end of the formation process. Although the interpretation from the density profiles seems inconsistent with the one inferred from the average column density distribution, it is not. For filaments exceeding the critical line-mass, the density distribution is described by an equilibrium profile only for radii below the scale height. The excess mass is at radii above the scale height resulting in a radial profile flatter than $r^{-4}$. As the Plummer profile only measures the radial dependence at radii above the scale height, such a description misses the equilibrium distribution in the center of the filament.

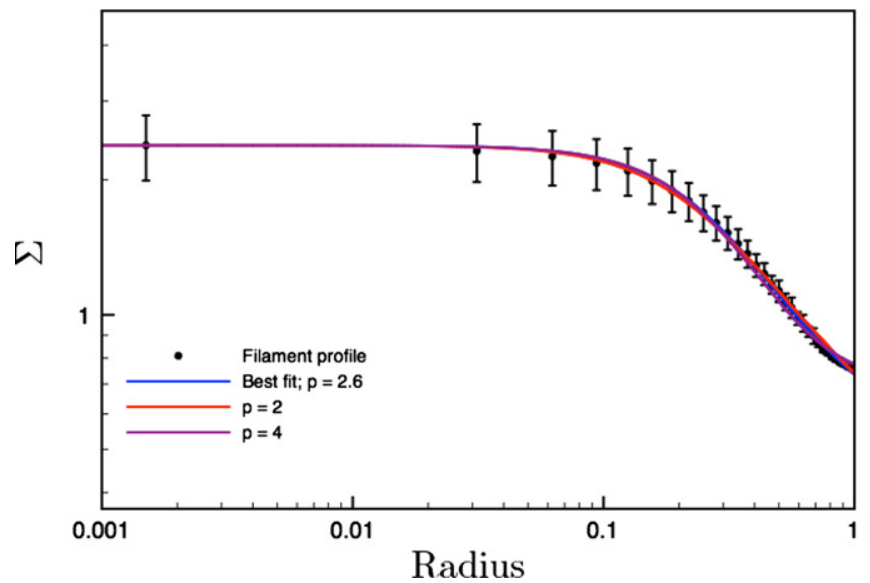

A more important result is that the filaments can be approximated by a hydrodynamical equilibrium without adjusting the sound speed (to take into account additional support provided by magnetic support). This suggests that the magnetic field is not important in setting the density distribution inside of the filaments. In filaments perpendicular to the magnetic field, gas flows along the field lines. Then magnetic pressure gradients are not generated and are thus much smaller than pressure (or density) gradients. In the quasi-hydrodynamical regime, filaments also form parallel to the magnetic fields. Because of magnetic flux conservation, a scaling of the magnetic field with density is established, i.e., $B \propto \rho$. The magnetic pressure, then, has a similar slope to that of the thermal pressure. However, as $\beta>1$, the magnetic pressure gradient is still much smaller than the thermal pressure gradient and does not contribute to the total pressure force, balancing self-gravity.

Dense core formation. The formation of dense cores is different in the two regimes. While cores form at the junctions of filaments in the weak magnetic regime, the cores in the strong magnetic regime form due to fragmentation of the filament. However, Inutsuka \& Miyama (1992) show that the filament only fragments if the growth rate of the unstable axisymmetric is shorter than the radial collapse time. This condition is only fulfilled if $m_{\text {line }} \approx m_{\text {crit }}$. For our initial conditions, the line-mass is much larger than the critical value, i.e., $m_{\text {line }}=1.9 m_{\text {crit }}$, and thus we do not expect cores to form within the filaments due to fragmentation. The condensations that we see in the filaments (see Figure 12) are actually a result of the initial conditions that break the uniformity of the layer.

\section{PERPENDICULAR MAGNETIC FIELD}

The previous section discusses the gravitational instability in a layer threaded by a parallel magnetic field. Here, we examine the fragmentation for a magnetic field perpendicular to the layer. Nakano \& Nakamura (1978) study the marginally stable modes of an isothermal layer with perpendicular magnetic field. They find that the layer is unstable to gravitational perturbations when $\Sigma_{0} / B_{0}>(\pi G)^{-1 / 2}$ or simply $\beta>1$, with the critical wavelength close to the hydrodynamical value. This means that, contrary to a parallel magnetic field, a perpendicular magnetic field can stabilize the layer as long as it is strong enough. Figure 18 indeed shows that perturbations in models with $\beta \leqslant 1$

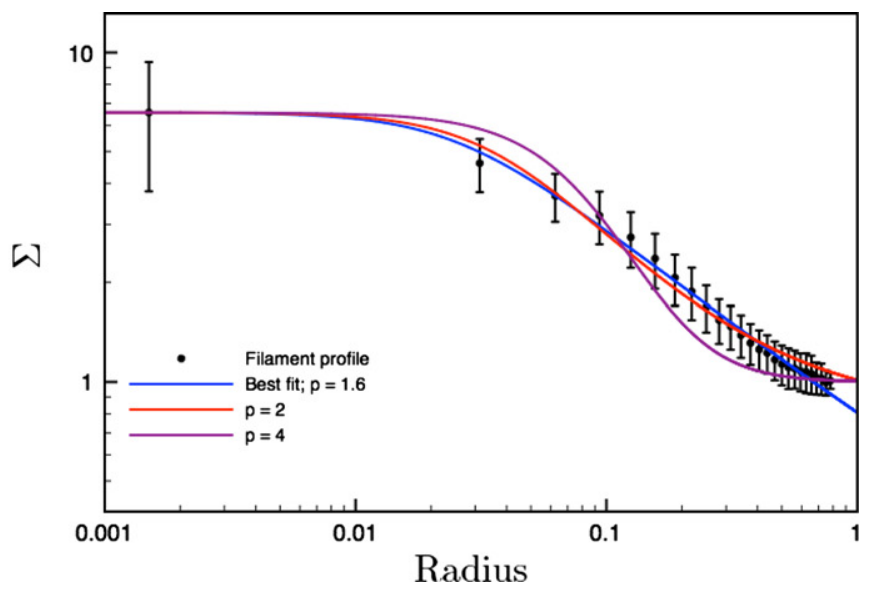

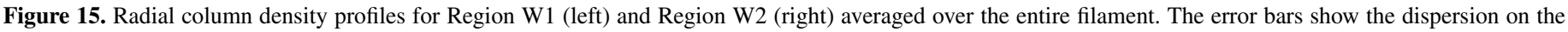

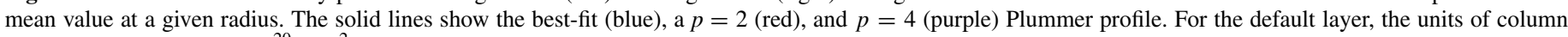
density and length are $5 \times 10^{20} \mathrm{~cm}^{-2}$ and $1 \mathrm{pc}$, respectively.

(A color version of this figure is available in the online journal.) 


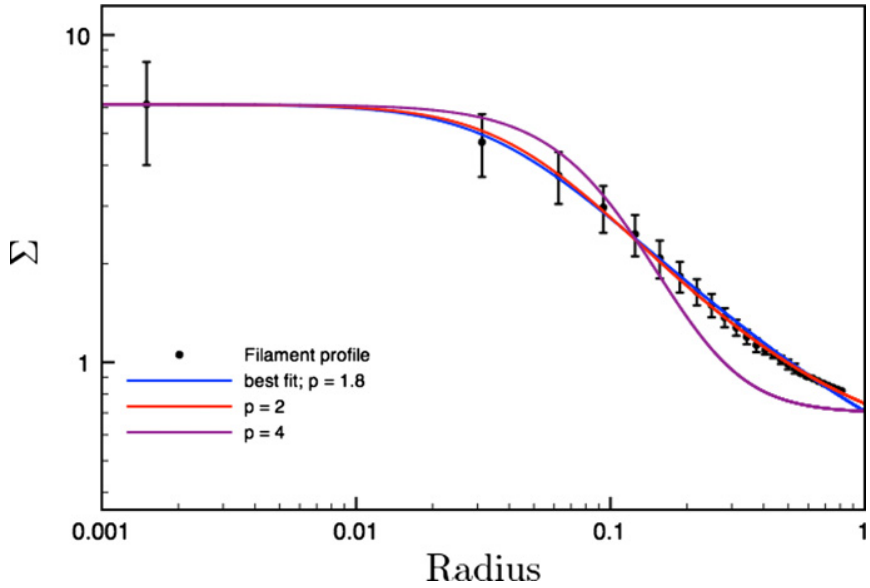

Figure 16. Average radial column density profile for Region S1 with the dispersion on the mean given by the error bars. The solid lines show the best-fit (blue), a $p=2$ (red) and $p=4$ (purple) Plummer profile. For the default layer, the units of column density and length are $5 \times 10^{20} \mathrm{~cm}^{-2}$ and $1 \mathrm{pc}$, respectively. (A color version of this figure is available in the online journal.)

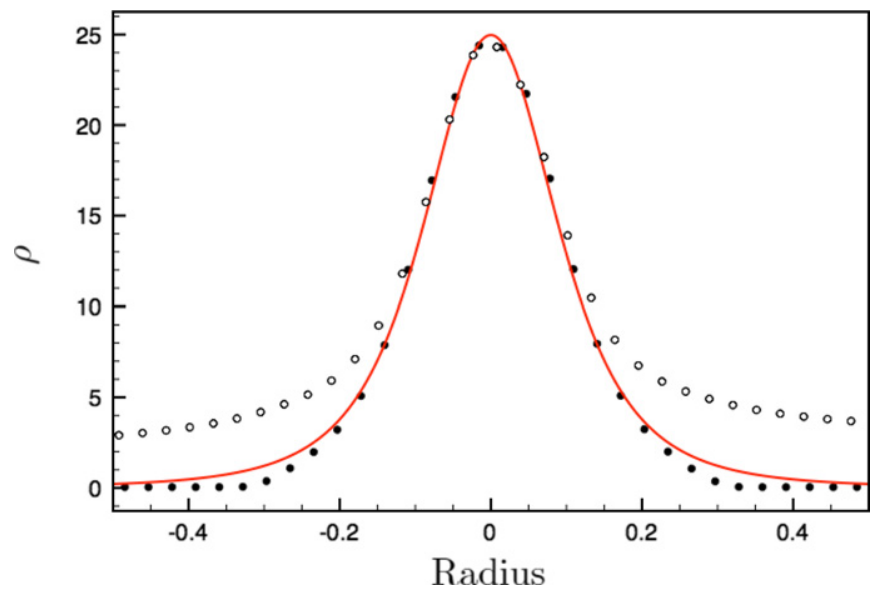

Figure 17. Density slice at $y=1$ for Region S1. The solid circles show the density along the $z$ axis and the open circles along the $x$ axis. The solid line is the profile for an Ostriker cylinder with $\rho_{c}=24.9$. In terms of the default layer, the length unit is $1 \mathrm{pc}$ and the density unit is $159.2 \mathrm{~cm}^{-3}$.

(A color version of this figure is available in the online journal.)

are damped. For these layers to become unstable, the magnetic support needs to disappear by, e.g., ambipolar diffusion (Kudoh et al. 2007; Kudoh \& Basu 2008).

Density structure and filaments. The $\beta=10$ model has a similar, though slightly slower, growth rate as the hydrodynamical model. This reduced growth rate is due to magnetic tension forces acting to straighten field lines (which are bended because the flow in the layer is perpendicular to the field lines). The mode associated with this growth still has a wavelength close to $\lambda_{\max }=4 \pi H$, as the resulting column density structure is very similar to the hydrodynamical model (see Figure 19). The filament ridges are actually in the same places.

Not only does the column density structure look very similar, the column density and density profiles of the filaments show the same properties as in the previous models (see Figure 20). The power index of the best-fit Plummer profile has a value of $\leqslant 2$, but, at the same time, the central density structure of the filaments is perfectly described by an equilibrium Schmid-Burgk profile. Again, this means that the magnetic field only contributes marginally to the dynamics. The plasma $\beta$ near the center of the filament decreases but is still above $\beta=1$.

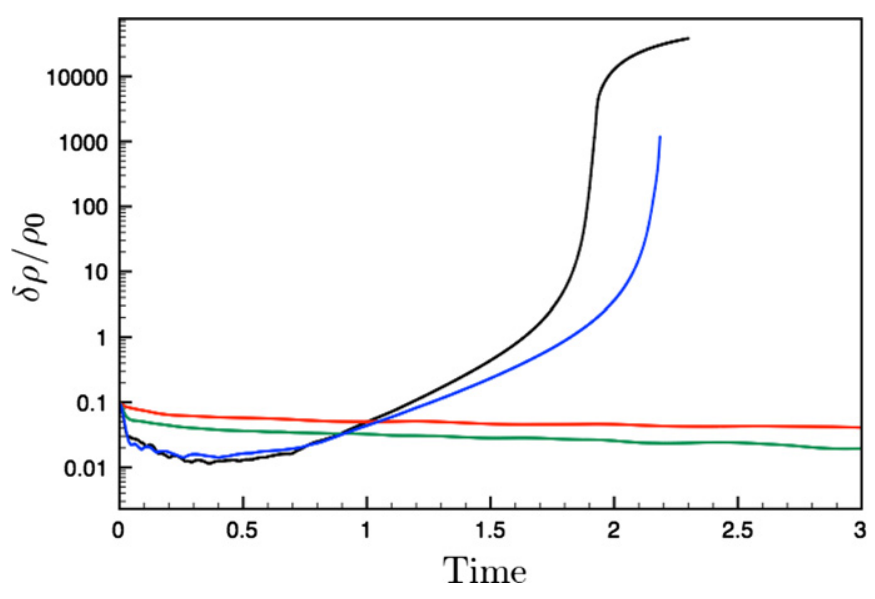

Figure 18. Similar to Figure 1, but for perpendicular magnetic fields. The solid lines represent a hydrodynamical (black), $\beta=10$ (blue), 1 (red), and 0.1 (green) model. The unit of time for our default layer is 4.9 Myr.

(A color version of this figure is available in the online journal.)

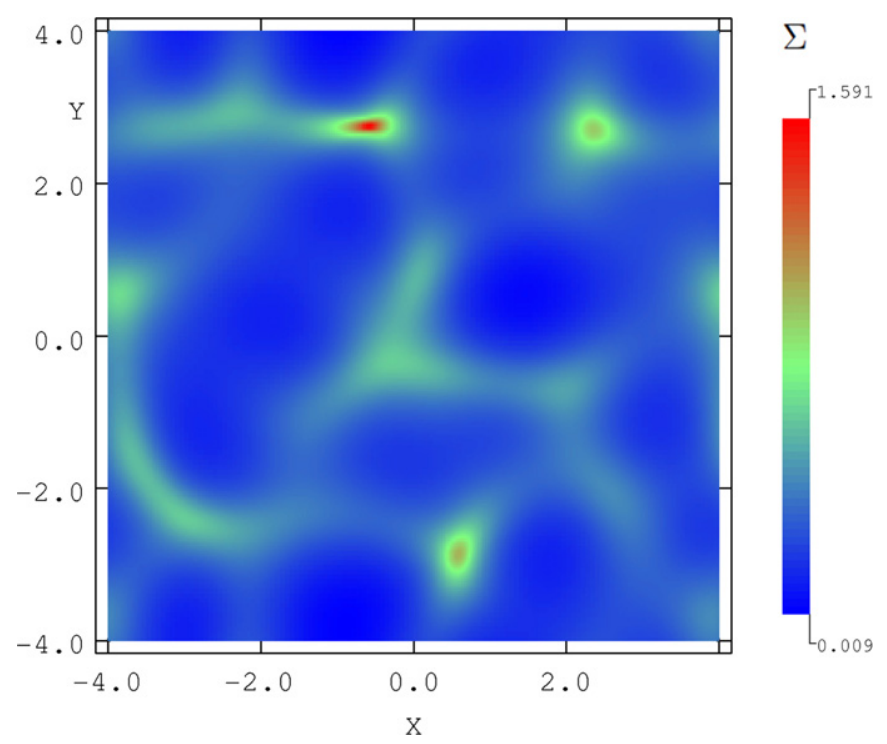

Figure 19. Column density structure along the $z$ axis at $t=2.15$ for a perpendicular magnetic field with $\beta=10$. For the default layer, the unit of column density is $5 \times 10^{20} \mathrm{~cm}^{-2}$.

(An animation and color version of this figure are available in the online journal.)

Dense cores. While the magnetic field does not influence filaments to any significant degree, the evolution of the cores is affected by them. Dense cores still arise at the junctions of the filaments, but the density distribution of the cores is very much axisymmetric. In the hydrodynamical model, gas is predominantly fed to the cores along the filaments and not isotropic as suggested here. Because of magnetic tension forces, gas in the vicinity of the cores flows directly toward the core instead of first to a filament and then toward the core. The former produces a magnetic field that is less 'twisted'. The axisymmetry of the dense core is also observed in the scaling relation of the magnetic field and density relation, i.e., $|B| \propto \rho^{2 / 3}$ (see Figure 21). Such a relation is obtained for an isotropically contracting sphere threaded by a frozen-in magnetic field. Although the magnetic energy increases more rapidly than the thermal pressure in the core (i.e., $\beta \propto \rho^{-1 / 3}$ ), the magnetic field is never strong enough to prevent collapse. The potential energy of a spherical core increases at the same rate as the magnetic energy during contraction. Initially, the gravitational energy 

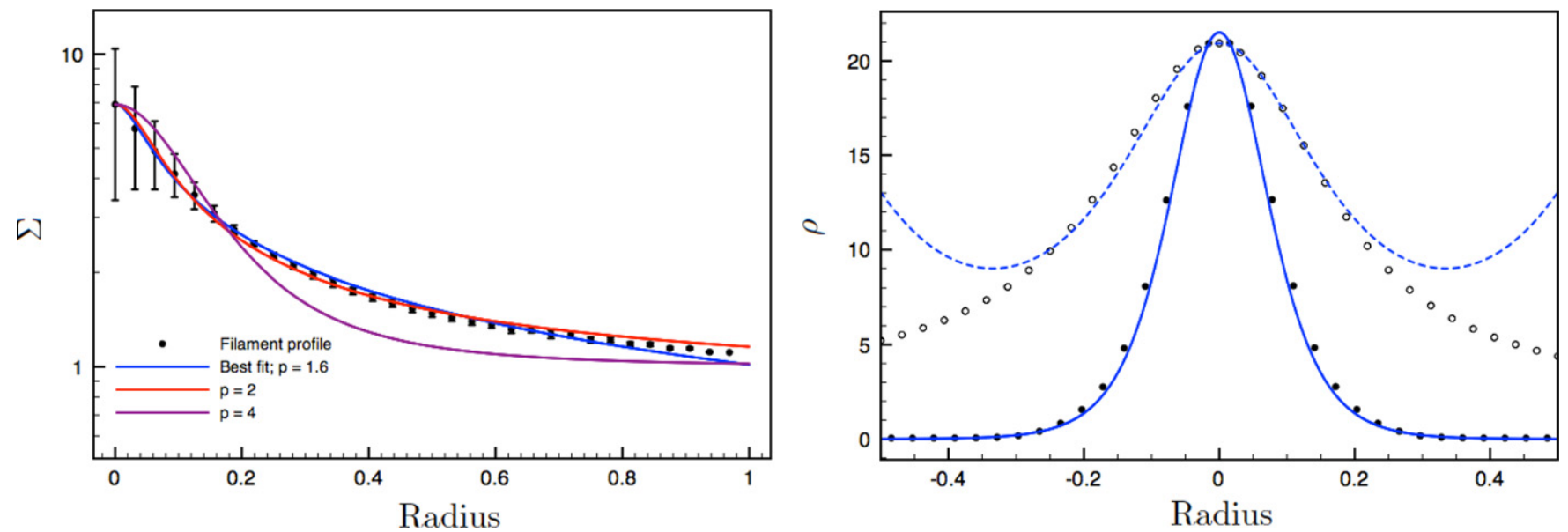

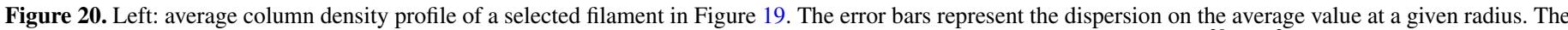

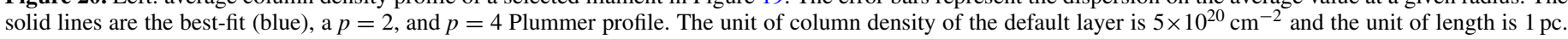

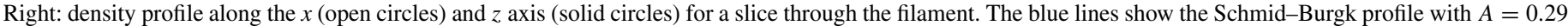
and $\rho_{c}=11.81$ along the $x$ (dashed) and $z$ (solid) axis. For the default layer, the unit of length is $159.2 \mathrm{~cm}^{-3}$.

(A color version of this figure is available in the online journal.)

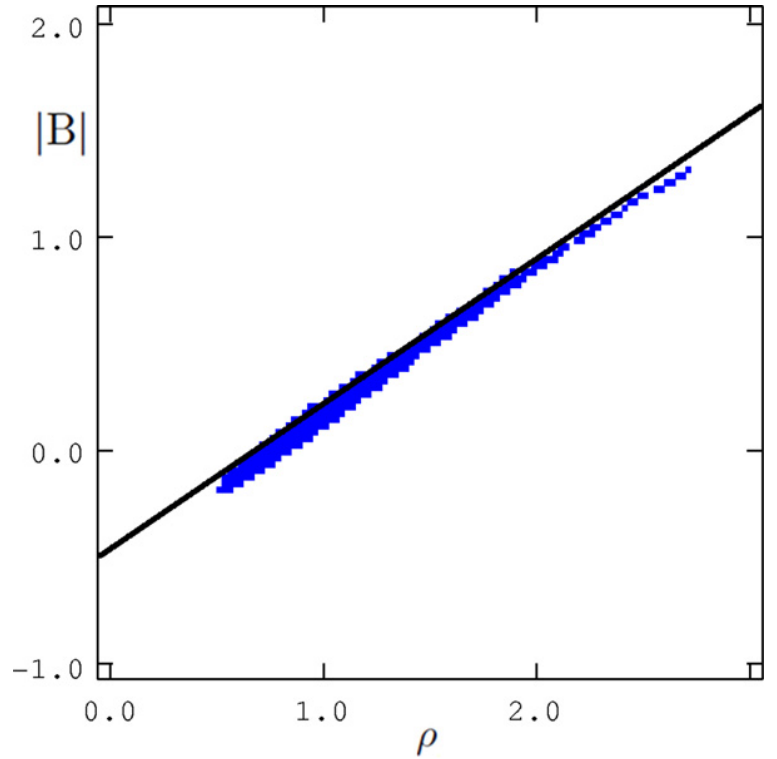

Figure 21. Log-log relation between the magnetic field magnitude and density at the midplane for the perpendicular $\beta=10$ model. The solid line shows the dependence for an isotropically contracting sphere. In terms of the default layer, the units of the magnetic field and density are given by $1.76 \mu \mathrm{G}$ and $152.9 \mathrm{~cm}^{-3}$. (A color version of this figure is available in the online journal.)

is larger than the magnetic energy and thus remains so during the subsequent evolution.

\section{COMPARISON WITH THE STAR-FORMING REGION, G14.225-0.506}

Recently, Busquet et al. (2013) reported Very Large Array observations in the $\mathrm{NH}_{3}$ of a massive star-forming complex, G14.225-0.506. The $\mathrm{NH}_{3}$ emission reveals a network of filaments that are aligned parallel in projection onto the plane of the sky (see Figure 22). The filaments appear to take two directions, one group is oriented at a position angle (P.A.) of $10^{\circ}$, and the other is at a P.A. of $60^{\circ}$. The averaged projected separation between the adjacent filaments are between $0.5 \mathrm{pc}$ and 1 pc. Polarimetric observations in the near infrared $\mathrm{H}$ band to- ward the northern section of the complex reveal magnetic field orientations perpendicular to the main axis of the filaments.

The fragmentation of a layer threaded with a strong parallel magnetic field reproduces the G14.225-0.506 filament network (see Figure 22). This allows us to infer the initial conditions of the initial, unperturbed layer. The separation of the adjacent filaments is given by $\lambda_{\max }=4 \pi H$ which results in a scale height of $0.04-0.08 \mathrm{pc}$ for the unperturbed layer. Then the initial surface density is $\Sigma=a^{2} /(\pi G H) \approx 1.5-3 \times 10^{-2} \mathrm{~g} \mathrm{~cm}^{-2}$ (assuming a sound speed of $0.2 \mathrm{~km} \mathrm{~s}^{-1}$ ). As the area covered by the filaments is $4.7 \times 8.7 \mathrm{pc}^{2}$, the unperturbed layer contains about $2900-5800 M_{\odot}$. This agrees well with the combined mass of $2600 M_{\odot}$ for the filaments in G14.225-0.506 (Busquet et al. 2013). Also, an estimate of the magnetic field strength can be made. For the strong parallel field model, $\beta<1$ and the required magnetic field is stronger than $12-25 \mu \mathrm{G}$. Zeeman measurements of the magnetic field indicate that these values are toward the high end of, but within, the observed range in magnetic field strengths (Crutcher 2012).

The initial conditions needed to produce the density structure of the filamentary complex G14.225-0.506 by gravitational instability in the presence of a strong magnetic field are not unrealistic. However, we cannot exclude any other formation process such as, e.g., colliding flows.

\section{DISCUSSION AND CONCLUSIONS}

In this paper, we have examined the gravitational instability in an isothermal equilibrium layer threaded by a magnetic field. Our main results are as follows.

1. An equilibrium isothermal layer threaded by parallel magnetic fields is always unstable for gravitational instabilities with wavelengths larger than $\lambda_{\text {crit }}=2 \pi H$. For perpendicular magnetic fields, instabilities only arise when $\beta>1$. The density structures of the unstable layer can be described by two different regimes, i.e., a hydrodynamical one and a strong magnetic one. The network structure of filaments can then be used to estimate the magnitude of the magnetic field. Parallel filaments indicate that the magnetic field is strong with $\beta \leqslant 1$ and that the field lines lie within the plane of the layer. On the other hand, a filament-hub structure implies that the magnetic field is weak with $\beta \gg 1$. 

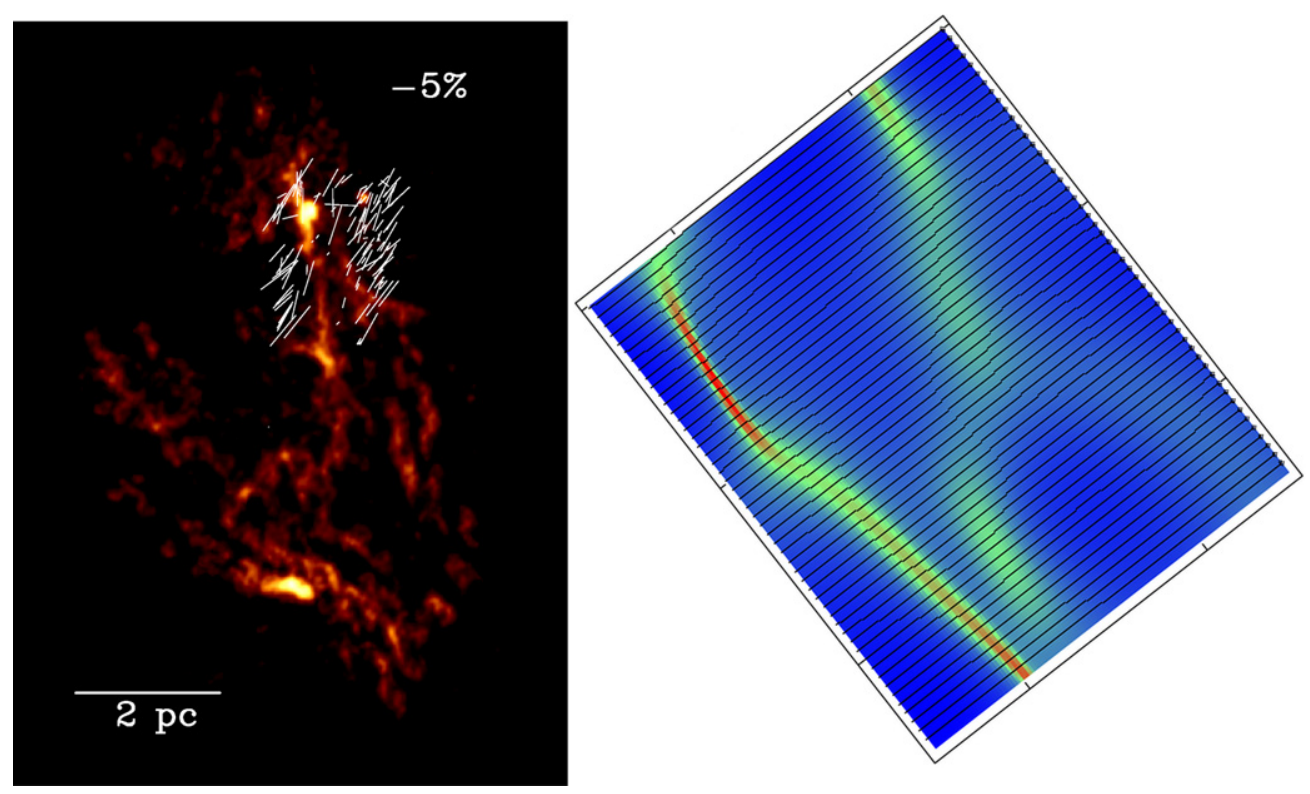

Figure 22. Left: $\mathrm{NH}_{3}$ emission map of the massive star-forming complex, G14.225-0.506 (Busquet et al. 2013). The white lines show the polarization vectors of the near-infrared (H-band) polarimetric observations (Busquet et al. 2013). Right: section of the right panel of Figure 12. The solid lines represent the mass-weighted magnetic field integrated along the $z$ axis.

(A color version of this figure is available in the online journal.)

In this case, there is no connection between filament axes and the magnetic field, i.e., the magnetic field can lie parallel or perpendicular along the filament axes.

2. The filaments that form have a line-mass that exceeds the critical value for axisymmetric collapse and thus cannot be in hydrostatic equilibrium. However, at any time during their evolution, the central regions of the filaments are described by an equilibrium density structure. Using a Schmid-Burgk (1967) density profile (Equation (5)), the evolution of a filament from a perturbation in the equilibrium layer $(A \approx 0)$ toward an Ostriker (1964) distribution $(A=1)$ is perfectly captured. Furthermore, because of the thermal support in the central region of the filament, the collapse time is much longer than the free-fall time scale (similar to the results of Inutsuka \& Miyama (1992) for collapsing cylinders).

Although the central region of filaments is well-described by equilibrium profiles, the distribution for radii above the scale height deviates from the equilibrium (as that region contains an excess of mass). The profile is then flatter than the expected power law of $r^{-4}$ and similar to the results from Herschel observations (e.g., Arzoumanian et al. 2011). An additional flattening of the profile is obtained by averaging the column density profile along a filament with a central density variation. An increase in the central density produces a larger central column density but also implies a smaller scale height (i.e., $R_{0} \propto \rho^{-1 / 2}$ ). Such a variation in the filament width is actually observed along the L1506 filament in the Taurus molecular complex (Ysard et al. 2013).

By studying colliding flows, Gomez \& VazquezSemadeni (2013) show that filaments also form due to dynamical and thermal instabilities in an initially sheet-like structure. While their filaments exhibit the flat column density profile of the observations and our simulations, their formation and evolution does not follow quasi-equilibrium states. Gomez \& Vazquez-Semadeni (2013) argue that the filaments are just long-lived, persistent features of the flow. However, the resolution of their simulation is not high enough to resolve the thermal scale height associated with the filaments (e.g., $\mathrm{H} \approx 0.3 \mathrm{pc}$ for their filament 1 , while the resolution is $\approx 0.17 \mathrm{pc}$ ). Without the proper resolution, thermal pressure forces cannot balance gravity numerically. We encounter the same problem when we analyze filaments with a central density that is too high $\left(\rho_{c}>150\right.$ in code units). We also recognize the difficulty in resolving the thermal scale height in turbulent simulations where the density contrasts rapidly fluctuate.

3. Our simulations do not reproduce the other property of filaments, i.e., a near constant FWHM of $\sim 0.1 \mathrm{pc}$. As the central density of the filaments increases, the scale height continues to decrease. As the scale height also depends on pressure support, i.e., $R_{0} \propto a$, the pressure support within the filament needs to increase as it is collapsing. Our simulations show that the magnetic fields are not able to provide this extra support.

However, Klessen \& Hennebelle (2010) show that turbulence can be driven by accretion. Interstellar filaments indeed show that the filament velocity dispersion increases with column density and exceeds the thermal sound speed if they have a line-mass above the critical value (Arzoumanian et al. 2013).

4. Different authors (e.g., Li et al. 2004; Banerjee et al. 2009; Collins et al. 2011) show that the magnetic field does not prevent the formation and collapse of dense structures in molecular clouds. However, these studies only consider a low level of magnetization. In the case of a strong magnetic field, simulations show that the field dominates turbulence and inhibits the gravitational collapse (e.g., Nakamura \& Li 2008; Basu \& Dapp 2010). The filaments in our simulations are purely hydrodynamical structures. This means that the magnetic field does not play a significant role in setting the shape of the filaments, even in a low-beta plasma. 
5. Dense cores form at the same time as the filaments. In the hydrodynamical regime, the cores arise at the junctions of the filaments. Mass accretion of the cores depends on the orientation of the magnetic field, i.e., for perpendicular fields the accretion is roughly isotropic, while, for parallel fields, much of the gas is fed along the filaments and thus highly directional. Such non-isotropic mass accretion drastically changes the observational signatures of dense core collapse (e.g., Smith et al. 2012, 2013). Enough mass is accreted for the cores to contract, but at rates slower than free-fall collapse. This again suggests that thermal pressure (and magnetic pressure to a lesser degree) is able to roughly balance against self-gravity.

Some caveats need to be taken into account when discussing these findings. Turbulent motions are ubiquitous in the ISM and it is unlikely that a thick, quiescent equilibrium layer forms out of such a medium. For example, simulations of colliding flows (e.g., Vázquez-Semadeni et al. 2003; Audit \& Hennebelle 2005; Heitsch et al. 2008) show that the shock-bounded layer is prone to dynamical instabilities, such as Kelvin-Helmholtz and Raleigh-Taylor instabilities, but also to thermal instabilities. However, Kudoh \& Basu (2011) show that, dense core profiles do not depend strongly on the initial velocity perturbations, though their formation time scales are shorter for larger velocity fluctuations.

For a thin isothermal layer, the nature of the gravitational instability changes and is very similar to an incompressible mode. Nagai et al. (1998) show that the density perturbations are then a result of a deformation of the boundary of the layer. When the layer is threaded by a strong magnetic field $(\beta<1)$, filaments no longer form perpendicular to the magnetic field lines but parallel to them. Furthermore, the line-mass of the filaments is smaller than the critical value. Then the filaments no longer collapse but are in equilibrium. It is then expected that the filament fragments along its axis into cores with equal spacing between them. We will study this possibility in a subsequent paper.

We thank Tom Hartquist, Sam Falle, and Phil Myers for useful discussions, Gemma Busquet for providing the observational data of the G14.225-0.506 molecular cloud and the anonymous referee for comments that improved the paper. S.V.L. acknowledges support from the SMA Postdoctoral Fellowship of the Smithsonian Astrophysical Observatory. The simulations for this work were run on the Smithsonian Institution High Performance Cluster (SI/HPC).

\section{REFERENCES}

Alves, J. F., Lada, C. J., \& Lada, E. A. 2001, Natur, 409, 159

André, P., Men'shchikov, A., Bontemps, S., et al. 2010, A\&A, 518, L102

Arzoumanian, D., André, P., Didelon, P., et al. 2011, A\&A, 529, L6

Arzoumanian, D., André, P., Peretto, N., \& Konyves, V. 2013, A\&A, 553, A119

Audit, E., \& Hennebelle, P. 2005, A\&A, 433, 1

Ballesteros-Paredes, J., Hartmann, L., \& Vázquez-Semadeni, E. 1999, ApJ, 527,285

Ballesteros-Paredes, J., Klessen, R. S., Mac Low, M.-M., \& Vazquez-Semadeni, E. 2007, in Protostars and Planets V, ed. B. Reipurth, D. Jewitt, \& K. Keil (Tucson, AZ: Univ. Arizona Press), 63

Ballesteros-Paredes, J., \& Mac Low, M.-M. 2002, ApJ, 570, 734
Banerjee, R., Vázquez-Semadeni, E., Hennebelle, P., \& Klessen, R. S. 2009, MNRAS, 398, 1082

Basu, S., \& Dapp, W. B. 2010, ApJ, 716, 427

Bonnor, W. B. 1956, MNRAS, 116, 351

Busquet, G., Zhang, Q., Palau, A., et al. 2013, ApJL, 764, L26

Collins, D. C., Padoan, P., Norman, M. L., \& Xu, H. 2011, ApJ, 731, 59

Crutcher, R. M. 2012, ARA\&A, 50, 29

Curry, C. L. 2000, ApJ, 541, 831

de Avillez, M. A., \& Breitschwerdt, D. 2005, A\&A, 436, 585

Dedner, A., Kemm, F., Kröner, D., et al. 2002, JCoPh, 175, 645

Elmegreen, B. G., \& Elmegreen, D. M. 1978, ApJ, 220, 1051

Falle, S. A. E. G. 1991, MNRAS, 250, 581

Falle, S., Hubber, D., Goodwin, S., \& Boley, A. 2012, in ASP Conf. Ser. 459, Numerical Modeling of Space Plasma Flows (ASTRONUM 2011), ed. N. V. Pogorelov, J. A. Font, E. Audit, \& G. P. Zank (San Francisco, CA: ASP), 298

Fiege, J. D., \& Pudritz, R. E. 2000, MNRAS, 311, 105

Fischera, J., \& Martin, P. G. 2012, A\&A, 542, A77

Galván-Madrid, R., Liu, H. B., Zhang, Z.-Y., et al. 2013, ApJ, 779, 121

Goldsmith, P., Heyer, M., Narayanan, G., et al. 2008, ApJ, 680, 428

Gomez, G. C., \& Vazquez-Semadeni, E. 2013, ApJ, submitted (arXiv:1308.6298)

Goodman, A. A., Bastien, P., Myers, P. C., \& Ménard, F. 1990, ApJ, 359, 363

Goodman, A. A., Jones, J. T., Lada, E. A., \& Myers, P. C. 1992, ApJ, 399, 108 Heitsch, F. 2013, ApJ, 769, 115

Heitsch, F., Hartmann, L. W., \& Burkert, A. 2008, ApJ, 683, 786

Inutsuka, S., \& Miyama, S. M. 1992, ApJ, 388, 392

Johnstone, D., \& Bally, J. 1999, ApJL, 510, L49

Keto, E., Rybicki, G. B., Bergin, E. A., \& Plume, R. 2004, ApJ, 613, 355

Kirk, H., Myers, P. C., Bourke, T. L., et al. 2013, ApJ, 766, 115

Klessen, R. S., \& Hennebelle, P. 2010, A\&A, 520, A17

Koyama, H., \& Inutsuka, S.-I. 2000, ApJ, 532, 980

Kudoh, T., \& Basu, S. 2008, ApJL, 679, L97

Kudoh, T., \& Basu, S. 2011, ApJ, 728, 123

Kudoh, T., Basu, S., Ogata, Y., \& Yabe, T. 2007, MNRAS, 380, 499

Ledoux, P. 1951, AnAp, 14, 438

Li, H.-b., Fang, M., Henning, T., \& Kainulainen, J. 2013, MNRAS, 436, 3707

Li, P. S., Norman, M. L., Mac Low, M.-M., \& Heitsch, F. 2004, ApJ, 605, 800

Lindeberg, T. 1998, International Journal of Computer Vision, 30, 117

Liu, H. B., Jiménez-Serra, I., Ho, P. T. P., et al. 2012, ApJ, 756, 10

Men'shchikov, A., André, P., Didelon, P., et al. 2010, A\&A, 518, L103

Miyama, S. M., Narita, S., \& Hayashi, C. 1987, PThPh, 78, 1273

Mizuno, A., Onishi, T., Yonekura, Y., et al. 1995, ApJ, 445, 161

Molinari, S., Bally, J., Noriega-Crespo, A., et al. 2011, ApJL, 735, L33

Myers, P. C. 1983, ApJ, 270, 105

Myers, P. C. 2009, ApJ, 700, 1609

Nagai, T., Inutsuka, S.-I., \& Miyama, S. M. 1998, ApJ, 506, 306

Nakajima, Y., \& Hanawa, T. 1996, ApJ, 467, 321

Nakamura, F., \& Li, Z.-Y. 2008, ApJ, 687, 354

Nakano, T., \& Nakamura, T. 1978, PASJ, 30, 671

Ostriker, J. 1964, ApJ, 140, 1056

Padoan, P., \& Nordlund, ̊.. 2002, ApJ, 576, 870

Palmeirim, P., André, P., Kirk, J., et al. 2013, A\&A, 550, A38

Peretto, N., André, P., Könyves, V., et al. 2012, A\&A, 541, A63

Pon, A., Toalá, J. A., Johnstone, D., et al. 2012, ApJ, 756, 145

Schmid-Burgk, J. 1967, ApJ, 149, 727

Schneider, S., \& Elmegreen, B. 1979, ApJS, 41, 87

Schneider, N., Csengeri, T., Hennemann, M., et al. 2012, A\&A, 540, L11

Smith, R. J., Shetty, R., Beuther, H., Klessen, R. S., \& Bonnell, I. A. 2013, ApJ, 771,24

Smith, R. J., Shetty, R., Stutz, A. M., \& Klessen, R. S. 2012, ApJ, 750, 64 Spitzer, L., Jr. 1942, ApJ, 95, 329

Tafalla, M., Myers, P. C., Caselli, P., \& Walmsley, C. M. 2004, A\&A, 416, 191 Toalá, J. A., Vázquez-Semadeni, E., \& Gómez, G. C. 2012, ApJ, 744, 190

Truelove, J. K., Klein, R. I., McKee, C. F., et al. 1997, ApJL, 489, L179 Umekawa, M., Matsumoto, R., Miyaji, S., \& Yoshida, T. 1999, PASJ, 51, 625 van Loo, S., Falle, S. A. E. G., \& Hartquist, T. W. 2006, MNRAS, 370, 975 van Loo, S., Falle, S. A. E. G., \& Hartquist, T. W. 2010, MNRAS, 406, 1260 van Loo, S., Falle, S. A. E. G., Hartquist, T. W., \& Moore, T. J. T. 2007, A\&A, 471,213

Vázquez-Semadeni, E., Ballesteros-Paredes, J., \& Klessen, R. S. 2003, ApJL, $585, \mathrm{~L} 131$

Ysard, N., Abergel, A., Ristorcelli, I., et al. 2013, A\&A, 559, A133 